\title{
Interventions for the treatment of uveitic macular edema: a systematic review and meta-analysis
}

This article was published in the following Dove Press journal:

Clinical Ophthalmology

10 June 2013

Number of times this article has been viewed

\author{
Rushmia Karim' \\ Evripidis Sykakis ${ }^{2}$ \\ Susan Lightman ${ }^{3}$ \\ Samantha Fraser-Bell ${ }^{4}$ \\ 'Faculty of Medicine, University of \\ Sydney, Camperdown, NSW, Australia; \\ 2Department of Ophthalmology, \\ Whipps Cross University Hospital, \\ ${ }^{3} \mathrm{UCL}$ Institute of Ophthalmology \\ and Moorfields Eye Hospital, London, \\ UK; ${ }^{4}$ University of Sydney, Clinical \\ Ophthalmology and Eye Health, \\ Sydney Adventist Hospital Clinical \\ School, Sydney, NSW, Australia
}

Background: Uveitic macular edema is the major cause of reduced vision in eyes with uveitis.

Objectives: To assess the effectiveness of interventions in the treatment of uveitic macular edema.

Search strategy: Cochrane Central Register of Controlled Trials, Medline, and Embase. There were no language or data restrictions in the search for trials. The databases were last searched on December 1, 2011. Reference lists of included trials were searched. Archives of Ophthalmology, Ophthalmology, Retina, the British Journal of Ophthalmology, and the New England Journal of Medicine were searched for clinical trials and reviews.

Selection criteria: Participants of any age and sex with any type of uveitic macular edema were included. Early, chronic, refractory, or secondary uveitic macular edema were included. We included trials that compared any interventions of any dose and duration, including comparison with another treatment, sham treatment, or no treatment.

Data collection and analysis: Best-corrected visual acuity and central macular thickness were the primary outcome measures. Secondary outcome data including adverse effects were collected

Conclusion: More results from randomized controlled trials with long follow-up periods are needed for interventions for uveitic macular edema to assist in determining the overall long-term benefit of different treatments. The only intervention with sufficiently robust randomized controlled trials for a meta-analysis was acetazolamide, which was shown to be ineffective in improving vision in eyes with uveitic macular edema, and is clinically now rarely used. Interventions showing promise in this disease include dexamethasone implants, immunomodulatory drugs and anti-vascular endothelial growth-factor agents. When macular edema has become refractory after multiple interventions, pars plana vitrectomy could be considered. The disease pathophysiology is uncertain and the course of disease unpredictable. As there are no clear guidelines from the literature, interventions should be tailored to the individual patient.

Keywords: uveitic macular edema, uveitis

\section{Introduction}

\section{Background}

Macular edema is one of the manifestations of uveitis leading to loss of central vision and reduced visual acuity. Macular edema most commonly occurs as a consequence of chronic intraocular inflammation. ${ }^{1}$ It is the most common cause of blindness and visual impairment in chronic uveitis patients occurring in up to one third of patients. ${ }^{2}$ Macular edema can be assessed clinically using slit-lamp biomicroscopy. Optical coherence
Correspondence: Rushmia Karim Faculty of Medicine, University of Sydney, Paramatta Rd, Camperdown, NSW 2006, Australia Email rushmiak@gmail.com 
tomography (OCT) can further aid in diagnosis and provide quantitative measures of central macular thickness. ${ }^{3}$ This is useful in order to monitor disease progress and efficacy of interventions, thus visual acuity and degree of macular edema (central macular thickness) are usually the primary end points studied. ${ }^{3}$

\section{Description of the condition}

Macular edema may persist even with successful control of the inflammatory response, especially in cases where uveitis has been chronic. Other causes of visual impairment in uveitis include glaucoma, optic nerve involvement, vitreous opacification from the inflammatory response, and cataract formation usually attributed to both the disease process and chronic steroid use. ${ }^{1}$

Interventions aim to resolve the inflammatory response in uveitis as well as treat or prevent the occurrence of macular edema. The exact pathology of macular edema is complicated and uncertain. The autoimmune theory is acceptable in patients who have been identified as genetically susceptible to uveitis. In these subjects, there is a generation of autoreactive $\mathrm{CD}^{+}$ $\mathrm{T}$ lymphocytes with an amplified cytokine response. Inner and outer blood-retinal barrier breakdown in the central retina due to prolonged or severe inflammation leads to macular edema. ${ }^{1}$ Blood-retinal barrier breakdown aids inflammatory cell migration. The retinal vascular endothelium changes with activation of adhesion molecules and lymphocytes. ${ }^{1}$

Macular edema causes an inflammatory response releasing mediators which damage the retinal pigment epithelium (RPE), leading to failure of its pump, metabolism, and waste system. ${ }^{1}$ This results in leakage of fluid into the retina, especially at the macula. The fluid is predominantly located in the outer plexiform layer, as seen on OCT scanning. ${ }^{4}$ Damage to the retinal vasculature also contributes to further disease and release of cytokines and other mediators. ${ }^{1}$ Chronic macular edema may lead to macular cysts and macular holes, resulting in nonreversible visual acuity loss. Development of an epiretinal membrane is also a consequence of chronic macular edema. Thus it is important to treat macular edema early. Further chronic macular edema may become more difficult to treat. Refractory macular edema usually occurs in patients with chronic or recurrent uveitis.

\section{Epidemiology}

Most of the epidemiological data is related to developed countries. The estimated annual incidence of uveitis is 17-52 cases per $100,000 .^{5}$ Prevalence is approximately $38-714$ per 100,000 . Uveitis is predominately a disease of adults between 20 and 60 years. ${ }^{5}$ The most common causes of noninfectious uveitis include Fuchs heterochromic iridocyclitis, human leukocyte antigen B27-associated uveitis, juvenile idiopathic arthritis-related uveitis, birdshot chorioretinopathy, sarcoidosis, multifocal choroiditis, Vogt-Koyanagi-Harada syndrome, serpiginous choroiditis, and Behçet's disease. ${ }^{5}$

\section{Treatment options}

\section{Corticosteroids}

Corticosteroids are the mainstay of treatment for uveitis because they target neutrophil transmigration and decrease cytokine production, but they have a multitude of other effects. The mechanism of action of steroids involves the inhibition of prostaglandin and leukotriene synthesis, as well as downregulation of cell adhesion and major histocompatibility molecules. ${ }^{6,7}$ Corticosteroids have been demonstrated to decrease the induction of vascular endothelial growth factor (VEGF) by proinflammatory mediators, such as plateletactivating factor, in a dose-dependent manner. ${ }^{8-10}$

\section{Systemic corticosteroids}

Oral prednisolone is often used to treat patients with significant vision-threatening uveitis. However, it is associated with systemic side effects. Long-term use of steroids can cause peptic ulceration, osteoporosis, and necrosis of the hip, weight gain, muscle weakness, hyperglycemia, and systemic hypertension, progression of glaucoma, and progression of cataracts. Less commonly, intravenous methyl prednisolone is used typically at much higher doses than that given orally, such as 500-1000 mg doses repeated over 2-3 days. ${ }^{1-13}$

\section{Periocular corticosteroid injections}

Steroids that are given locally by the periocular route are thought to be more advantageous than topical steroids for cystoid macular edema (CME), due to the proximity of drug to the macula (the site of action), and a higher concentration of drug is available in vitreous, retina, and choroid. ${ }^{11}$ Potential complications include globe perforation, ptosis, subdermal fat atrophy, extraocular muscle paresis, optic nerve injury, retinal and choroidal vascular occlusion, and cutaneous hypopigmentation. Forms of periocular injections include subconjunctival, orbital floor, and sub-Tenon. ${ }^{14}$

Venkatesh et al describe ways of injecting periocular corticosteroids: the Smith and Nozik method and the cannula method. ${ }^{14}$

The Smith and Nozik method involves the patient looking inferonasally whilst the conjunctiva is lifted with serrated forceps. A steroid-filled syringe is advanced with the bevel 
facing towards the globe, superotemporally along the curve of the globe. The needle is advanced until the hub touches the conjunctiva. The plunger is slightly withdrawn to rule out injecting steroids within a vessel. ${ }^{14}$

In the cannula method, a wire speculum is placed and the patient is asked to look inferonasally. Conjunctiva along with Tenon is lifted approximately $10 \mathrm{~mm}$ away from the limbus using blunt serrated forceps. A 22-gauge cannula is inserted and advanced $3 \mathrm{~mm}$ within the episcleral space. The cannula is advanced about $12-14 \mathrm{~mm}$ in the sub-Tenon space with the stylet withdrawn. The syringe is then loaded with the steroid and injected. ${ }^{14}$

\section{Intravitreal triamcinolone acetonide (IVTA)}

IVTA allows high steroid concentration to act locally for maximal effect and duration. It has been used to treat macular edema from a variety of other etiologies, including retinal vein occlusion, diabetic retinopathy, pseudophakic $\mathrm{CME}$, and exudative macular degeneration. ${ }^{9,10,15,16}$ Although local side effects are relatively common, such as increased intraocular pressure and cataract progression similar to periocular steroids, the systemic side effects are minimal..$^{9,10,17}$

\section{Corticosteroid implants}

Sustained corticosteroid-release implants have been developed for injection into the vitreous with a longer duration than IVTA. The Retisert (fluocinolone acetone; Bausch and Lomb Place, Rochester, NY, USA) implant is an example of a nonbiodegradable implant, whereas the Ozurdex (dexamethasone; Allergan, Irvine, CA, USA) is biodegradable.

\section{Dexamethasone implant}

The dexamethasone implant Ozurdex uses a Novadur ${ }^{\circledR}$ (Allergan) solid polymer-delivery system, in which biodegradable material is combined with dexamethasone to form a rod-shaped implant. It is injected into the vitreous using an injector. Dexamethasone is then released over 3-6 months. It can be inserted in the clinic, in contrast to nonbiodegradable implants, which require a surgical procedure in the operating theater. ${ }^{18,19}$ Ozurdex has currently been licensed in the US and UK for use in CME caused by branch and central retinal vein occlusions. It is licensed in the US for posterior uveitis. ${ }^{18,19}$

\section{Fluocinolone acetone implant}

Retisert (fluocinolone acetone) is a long-term, slow-release intravitreal implant that was based on those used to deliver ganciclovir to patients with cytomegalovirus retinitis. The implant itself is a $1.5 \mathrm{~mm}$ tablet with overall dimensions of
$3 \times 2 \times 5 \mathrm{~mm} \cdot{ }^{19,20}$ Fluocinolone acetonide has high potency, low solubility, and a very short duration of action in the systemic circulation, enabling the steroid pellet to be small and reducing the risk of systemic side effects. The implant is surgically placed into the vitreous cavity. Pharmacokinetic studies in rabbits have demonstrated the delivery of constant levels of the corticosteroid to the posterior pole. Although there is a reduction in systemic side effects, there are significant local side effects, including increased intraocular pressure requiring filtration surgery and cataract progression. ${ }^{21-25}$

\section{Acetazolamide and somatostatin analogs}

Acetazolamide is a carbonic anhydrase inhibitor. Carbonic anhydrase catalyzes the hydration of carbon dioxide to bicarbonate, which dissociates to form hydrogen ions and bicarbonate. One subtype of carbonic anhydrase is isoenzyme IV, which is thought to be a membrane-bound fraction found in the apical region of the RPE cell. Inhibition of carbonic anhydrase reduces aqueous production and possibly fluid leaking from the RPE. ${ }^{26}$ Acetazolamide has been shown to increase the rate of fluorescein clearance from the vitreous and the rate of subretinal fluid resorption in animals. ${ }^{26}$

Octreotide is a somatostatin analog. It is a potent inhibitor of the release of growth hormone and other hormones. It is most commonly used for the treatment of acromegaly, carcinoid tumors, and vasoactive intestinal polypeptidesecreting tumors. ${ }^{27}$

Studies suggest that somatostatin is synthesized in the retina by the RPE housing receptors sst1, sst2, and sst5. ${ }^{27,28}$ Somatostatin analog may inhibit proliferation of human retinal endothelial cells and aid restoration of the inner blood-retinal barrier, which breaks down in posterior uveitis, leading to macular edema. Given the receptors are housed in the RPE, somatostatin may play a role in fluid and iontransport balance. Somatostatin may also inhibit the immune response and have a role in controlling inflammation. Hence, octreotide has been hypothesized as a potential treatment for uveitic macular edema. ${ }^{27,28}$

\section{Anti-VEGF treatment}

Monoclonal antibodies against VEGF were first developed as an intravenous treatment for metastatic colorectal cancer. ${ }^{29,30}$ Bevacizumab is a full-length humanized monoclonal antibody against VEGF, meaning it binds to all subtypes of VEGF. Pegaptanib is a synthesized anti-VEGF aptamer of a single ribonucleic acid strand that specifically targets VEGF-165 and binds only to it. Aptamers are oligonucle- 
otide ligands that are selected for high-affinity binding to molecular targets. Ranibizumab (rhuFab-VEGF) is an antibody fragment which neutralizes all VEGF isoforms and bioactive fragments. ${ }^{3}$

VEGF has an important role in angiogenesis (ie, the migration and mitosis of endothelial cells), upregulating methane monooxygenase and $\alpha v \beta 3$ activity, and the creation of blood-vessel lumen and fenestrations. ${ }^{3}$ As seen in preclinical models, VEGF has been shown to facilitate survival of existing vessels, contribute to vascular abnormalities (eg, tortuousness and hyperpermeability) that may impede effective delivery of antitumor compounds, and stimulate new vessel growth. VEGF has been shown to be an endothelial cell-specific mitogen, an angiogenic inducer, and is also known to increase retinal vessel permeability. ${ }^{3}$

In 2001, Fine et al authored a paper that investigated an association between CME and VEGF concentration in the aqueous humor and plasma of uveitis patients. This crosssectional study measured VEGF concentrations by enzymelinked immunosorbent assays in the aqueous humor in uveitic and healthy patients. Uveitis patients had higher aqueous humor concentration and lower plasma VEGF levels than healthy volunteers $(P=0.044$ and $P=0.002$, respectively). Thus anti-VEGF may be useful in the treatment of uveitic macular edema. ${ }^{31}$

Safety studies of intravitreal injections of anti-VEFG looking at rates of endophthalmitis and rhegmatogenous retinal detachments have been performed. These showed low incidence rates of $0.02 \%$ and $0.013 \%$, respectively. ${ }^{32,33}$

\section{Immunomodulatory drugs}

Immunomodulatory drugs have been explored in the management of uveitis complicated by macular edema as steroidsparing agents to reduce side effects from corticosteroids. ${ }^{34}$ Immunomodulatory drugs including mycophenolate mofetil, methotrexate, T-cell inhibitors like cyclosporine, interferons (IFNs), and anti-tumor necrosis factor (TNF)- $\alpha$.

\section{Biological agents - adalimumab and infliximab}

TNF- $\alpha$ is a key proinflammatory cytokine, and high intraocular levels have been found in experimental autoimmune and human uveitis. Biological agents including anti-TNF- $\alpha$ are attractive treatment options because they offer a more targeted suppression of immune effector responses.

Adalimumab is a fully human antibody. ${ }^{12}$ Infliximab is also a monoclonal antibody against TNF- $\alpha$. Infliximab is a chimeric antibody, ie, it has a murine component. ${ }^{35}$ In serum, aqueous humor, and vitreous of patients with uveitis, the intercellular adhesion molecule 1 (ICAM-1) levels significantly increase. ICAM may play a role in the regulation of vascular permeability through the leukocyte-endothelium interaction. Efalizumab is an inhibitor of ICAM that inhibits binding of lymphocyte function-associated antigen. This in turn could assist in uveitis that may be caused by disruption of the blood-retinal barrier with increased vascular permeability and increased ICAM levels. ${ }^{12,35-37}$

\section{Interferon- $\alpha$}

IFN- $\alpha$ is a cytokine belonging to type 1 IFNs. It can be produced by virtually all somatic cells after viral infection and exerts antiviral, antiproliferative, antiangiogenic, and immunomodulatory effects. IFNs influence both innate and adaptive immune responses and play a role in the defense against viral infections and tumor growth. In autoimmune diseases, IFNs appear as double agents, involved in both supportive and suppressive action. ${ }^{38-42}$

\section{Mycophenolate mofetil}

Mycophenolate mofetil blocks the de novo pathway of purine synthesis, which is selective for T lymphocytes. This interrupts DNA replication. ${ }^{26}$ It has been used to treat rheumatoid arthritis, pemphigus vulgaris, and psoriasis, showing a downregulation in specific cytokines that contribute to edema. Mycophenolate has been used to treat uveitis, especially birdshot chorioretinopathy and its associated macular edema. A few reports have been published on the treatment of uveitis, including a case series of patients with uveitis unresponsive to traditional immunosuppressants and retrospective reviews. ${ }^{44-48}$ More recent reports reiterate safety of long-term treatment with mycophenolate for noninfectious uveitis. Disease stability was seen in $72 \%-84.6 \%$ of patients after 2 years. ${ }^{44,45}$ This has been shown to be effective in pediatric patients as well. ${ }^{49}$

\section{Methotrexate}

Methotrexate inhibits dihydrofolic acid reductase. Dihydrofolates must be reduced to tetrahydrofolates by this enzyme before they can be utilized as carriers of one-carbon groups in the synthesis of purine nucleotides and thymidylate. ${ }^{26}$ Therefore, methotrexate interferes with DNA synthesis, repair, and cellular replication. Methotrexate can be given orally, intramuscularly, subcutaneously, intravenously, and intra-arterially. Intravitreal methotrexate has been used as first-line therapy in primary vitreoretinal lymphoma (associated with primary 
central nervous system lymphoma), with a good safety profile. It is used systemically in uveitis as a steroid-sparing agent. ${ }^{50,51}$ Only a few papers have been written regarding intraocular methotrexate for uveitic macular edema. ${ }^{51-53}$

\section{Pars plana vitrectomy}

Vitrectomy is an operation to remove the vitreous humor of the eye. Pars plana vitrectomy removes the posterior vitreous with a view of the posterior pole. Vitrectomy is an invasive operation with the possibility of significant intraoperative and postoperative complications. These include hemorrhage, endophthalmitis, retinal detachment, macular hole, and glaucoma and cataract formation. ${ }^{54-56}$ It also increases the clearance of medications injected into the vitreous, such as IVTA and anti-VEGF agents.

\section{Protocol}

\section{Why it is important to do this review}

The visual prognosis in inflammatory macular edema is poor in a substantial proportion of patients, with no clear treatment for refractory macular edema. There is a diverse group of interventions used by clinicians internationally, including off label interventions such as IVTA and anti-VEGF agents. Numerous studies have been and are being published, but their methodological quality has been variable. This systematic review was therefore designed to investigate the effectiveness and safety of all treatments of uveitic macular edema.

\section{Objectives}

The objective of this review is to assess the efficacy of all interventions in the treatment of uveitic macular edema. Tables 1 and 2 show inclusion and exclusion criteria for the studies considered for this review.

\section{Data sources}

The Cochrane Central Register of Controlled Trials (Central) (which contains the Cochrane Eyes and Vision Group Trials Register) in the Cochrane Library, Medline, and Embase were searched. There were no language or data restrictions in the search for trials. The databases were last searched on October 1, 2011. Reference lists of included trials were searched. The Australian National Health and Medical Research Council guidelines for macular edema references were searched. Archives of Ophthalmology, Ophthalmology, Retina, the British Journal of Ophthalmology, and the New England Journal of Medicine were searched for clinical
Table I Inclusion criteria for considering studies for this review

\begin{tabular}{|c|c|}
\hline Types of studies & Randomized controlled trials \\
\hline Participants & $\begin{array}{l}\text { Included trials that have enrolled participants of } \\
\text { any age and sex with any type of uveitic macular } \\
\text { edema, ie, including early, chronic, refractory, or } \\
\text { secondary uveitic macular edema }\end{array}$ \\
\hline Interventions & $\begin{array}{l}\text { Included trials that compared any interventions } \\
\text { of any dose and duration, including sham or no } \\
\text { treatment }\end{array}$ \\
\hline $\begin{array}{l}\text { Outcome } \\
\text { measures }\end{array}$ & $\begin{array}{l}\text { Primary outcome - BCVA: the difference in BCVA } \\
\text { as continuous data (converted in logMAR) } \\
\text { One or more lines of improvement from baseline } \\
\text { (ETDRS, Snellen, or logMAR equivalent) } \\
\text { Central macular thickness: retinal thickness } \\
\text { from baseline as measured by ocular coherence } \\
\text { tomography }\end{array}$ \\
\hline $\begin{array}{l}\text { Secondary } \\
\text { outcomes }\end{array}$ & $\begin{array}{l}\text { Anatomical measures: one or more grade } \\
\text { reduction of macular edema } \\
\text { Presence of edema via direct fundoscopy } \\
\text { Fluorescein angiography leakage } \\
\text { Vitreous haze: posterior vitreous penetration } \\
\text { ratios and mid-vitreous penetration ratios }\end{array}$ \\
\hline Adverse effects & $\begin{array}{l}\text { Ocular hypertension } \\
\text { Anterior chamber reaction } \\
\text { Lens opacity progression (cataract formation) } \\
\text { Endophthalmitis } \\
\text { Ocular irritation } \\
\text { Fibrous proliferation } \\
\text { Iris or retinal neovascularization } \\
\text { Retinal detachment } \\
\text { Reduction in visual acuity and blindness } \\
\text { Systemic side effects } \\
\text { Cognitive effects } \\
\text { Death }\end{array}$ \\
\hline $\begin{array}{l}\text { Quality-of-life } \\
\text { measures }\end{array}$ & No data \\
\hline Economic data & No data \\
\hline
\end{tabular}

Abbreviations: BCVA, best-corrected visual acuity; logMAR, logarithm of minimum angle of resolution; ETDRS, Early Treatment Diabetic Retinopathy Study.

trials and reviews. Hand-searching of references and their associated clinical trials was conducted. Unpublished clinical trials and those in progress were searched using clinical trials repositories, including the National Institutes of Health repository, the Current Controlled Trials repository,

Table 2 Exclusion criteria for considering studies for this review

\begin{tabular}{ll}
\hline $\begin{array}{l}\text { Exclusion } \\
\text { criteria }\end{array}$ & RCTs for interventions for uveitis with no mention \\
of macular edema were excluded in the analysis \\
RCTs of interventions for macular edema due to other \\
causes than uveitic macular edema were excluded \\
Full text of every study was reviewed and discussed \\
Studies that were not an RCT were excluded in the \\
analysis but discussed in the review
\end{tabular}

Abbreviation: RCT, randomized controlled trial. 
and the National Research Register Repository. Authors of unpublished closed trials were contacted for initial results. For full search details, see Supplementary materials.

\section{Criteria for considering studies for this review \\ Selection of studies}

Screening of titles and abstracts resulting from electronic and manual searches were reviewed. Abstracts were classified as relevant, potentially relevant, or not relevant for this review. Full copies of abstracts were obtained for relevant and potentially relevant reviews. Abstracts and full reviews were read to determine inclusion. Only randomized clinical trials were eligible. Study findings were in concordance with the Quorom statement. Figure 1 illustrates this selection of studies with a flow diagram. For full details of excluded trials, see Supplementary materials.

\section{Methods}

\section{Data extraction and management}

Table 3 illustrates extracted data for the primary and secondary outcomes for this review.

\section{Data synthesis}

Best-corrected visual acuity (BCVA) and central macular thickness (CMT), the primary outcome variables, were expressed as continuous variables. Standard deviations were calculated using actual $P$-values obtained from $t$-tests quoted by Cochrane. For every study, we calculated the mean difference for the primary outcome BCVA, logarithm of the minimum angle of resolution, and the CMT using 95\% confidence intervals. The outcome measures were pooled by use of the fixed-effect model, as there were only two trials to compare for one intervention in the meta-analysis.

Heterogeneity was calculated using Cochrane's $Q$ statistic and quantified using the $I^{2}$ statistic. These indicated the proportion of variability across studies due to heterogeneity, rather than sample error. Despite a high $I^{2}$, results were pooled, as examination of these studies on a Forest plot indicated that the individual trial results were consistent in the direction of the effect (ie, the mean difference and confidence intervals largely fell on one side of the null line).

Clinical heterogeneity was present between the studies in relation to dosage used. Follow-up varied from 4 to 12 weeks. Despite clinical heterogeneity, trials were pooled and overall

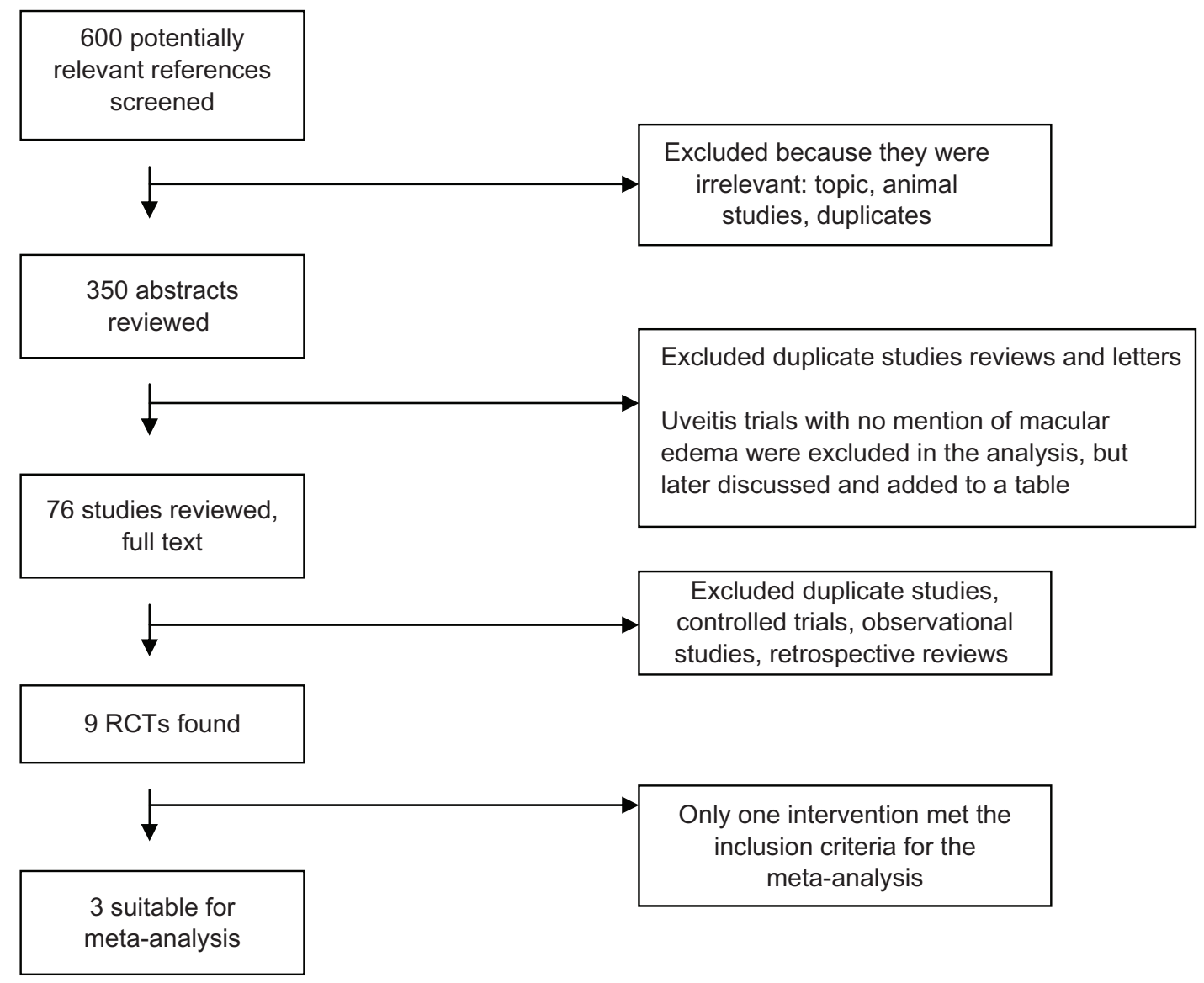

Figure I Study selection flow diagram.

Abbreviation: RCT, randomized clinical trial. 
Table 3 Extracted data (see summary of excluded and included studies and future studies in Supplementary materials)

\begin{tabular}{|c|c|}
\hline Participant & Total number \\
\hline \multirow[t]{10}{*}{ characteristics } & Sex \\
\hline & Age \\
\hline & Country \\
\hline & Type of uveitic macular edema \\
\hline & Diagnostic criteria \\
\hline & Baseline visual acuity or change in BCVA \\
\hline & Visual fields \\
\hline & Fluorescein angiography \\
\hline & OCT-determined thickness of central retinal thickness \\
\hline & Patient inclusion and exclusion criteria \\
\hline \multirow[t]{5}{*}{ Intervention } & Agent \\
\hline & Dose \\
\hline & Timing of first dose in relation to diagnosis \\
\hline & Delivery route \\
\hline & Frequency and treatment length \\
\hline Study and & Study design \\
\hline \multirow[t]{5}{*}{ methodology } & Trial identifiers \\
\hline & Study size \\
\hline & Randomization \\
\hline & Masking, allocation concealment \\
\hline & Duration of each study \\
\hline Primary & BCVA \\
\hline \multirow[t]{2}{*}{ outcomes } & Change in visual acuity \\
\hline & OCT \\
\hline Secondary & Retinal thickness from baseline, as measured by OCT \\
\hline \multirow[t]{3}{*}{ outcomes } & Anatomical measures \\
\hline & Presence of edema via direct fundoscopy \\
\hline & Fluorescein angiography leakage \\
\hline \multirow[t]{12}{*}{ Adverse effects } & Ocular and systemic toxicity \\
\hline & Ocular hypertension \\
\hline & Anterior chamber reaction \\
\hline & Lens opacity progression \\
\hline & Endophthalmitis \\
\hline & Ocular irritation \\
\hline & Fibrous proliferation \\
\hline & Iris or retinal neovascularization \\
\hline & Retinal detachment \\
\hline & Reduction in visual acuity and blindness \\
\hline & Cognitive effects \\
\hline & Death \\
\hline \multirow[t]{2}{*}{ Additional data } & Economic data, quality-of-life data \\
\hline & Treatment compliance and losses to follow-up \\
\hline \multirow[t]{4}{*}{ Missing data } & Authors contacted \\
\hline & Data has been entered in Review Manager (RevMan) \\
\hline & computer program, version 5.1 \\
\hline & Fixed-effect models used \\
\hline Data collection & Microsoft Excel spreadsheet \\
\hline
\end{tabular}

Abbreviations: BCVA, best-corrected visual acuity; OCT, optical coherence tomography.

efficacy from dose or follow-up of intervention were assessed in the objectives.

Subgroup analysis was not performed due to the limited trials. Characteristics of age, sex ratios, and baseline visual acuity were similar across all trials; however, variability in trial quality and intervention type, dose, and timing of administration varied. Table 4 highlights the characteristics of the included studies. Characteristics of both included and excluded studies are outlined in Supplementary materials.

Asymmetry assessment of the funnel plot was conducted for publication bias only to showcase the sample interventions. It cannot be used to assess publication bias, due to the limited number of trials. In future analyses, asymmetry of the funnel plot will be used to identify publication bias if at least seven studies are used. In the case of missing data, efforts to contact authors were made. Data was entered in Review Manager 5 (Cochrane, San Francisco, CA, USA), and fixed-effect models were used. Assessment of risk of bias in included studies has been considered. The following parameters were assessed: randomization process, allocation concealment, and masking of participants and investigators. Table 4 offers a summary of included study characteristics for trial qualityassessment score.

\section{Literature search synthesis Description of studies}

The electronic search, conducted in July 2011 and October 2011, resulted in 600 abstracts, of which 350 abstracts were reviewed. Of those, 76 full texts were read and nine found to be eligible. The reference lists of all nine randomized controlled trials (RCTs) were searched for relevant articles. Full texts of the major reviews found were read and their reference lists searched. Studies that were excluded are listed in Supplementary materials. To our knowledge, there are more than ten ongoing clinical trials related to uveitic macular edema. Authors and trial groups were contacted, and preliminary data were unable to be obtained for most of the studies. For one study, unpublished results were obtained. See Table 4 for a summary of included study characteristics.

\section{Types of interventions}

The interventions with results are listed below:

- periocular corticosteroid injections

- IVTA application

- corticosteroid implants

- anti-VEGF treatment

- immunomodulatory drugs, including IFNs and anti-tumor necrosis factor- $\alpha$, and acetazolamide, and somatostatin analogs

- pars plana vitrectomy

- other: Vitamin E and macular grid laser. 


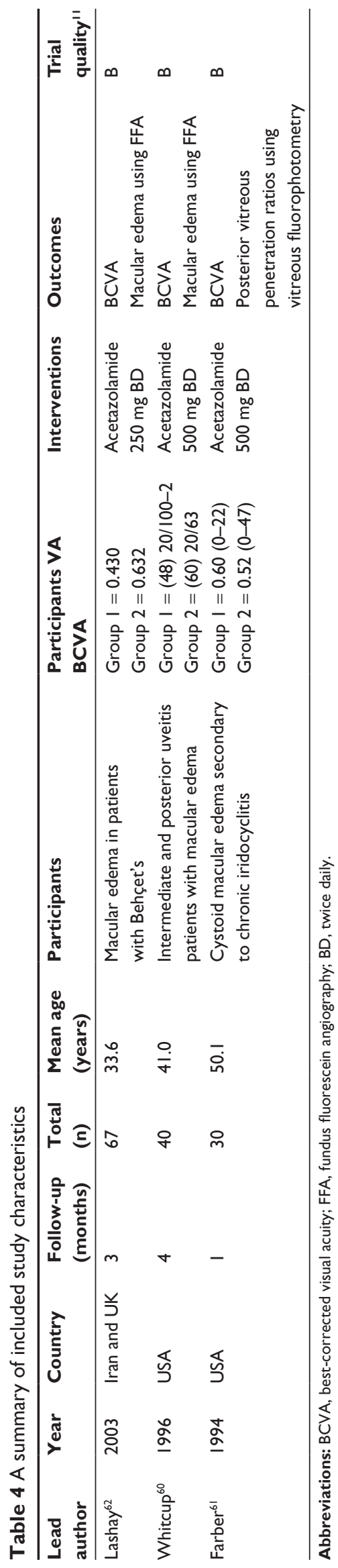

\section{Types of studies}

All interventional studies for uveitic macular edema were included. A description of each of these studies is presented in Supplementary materials and summarized in Table S1. All RCTs were included in an analysis.

\section{Types of participants}

There were only nine RCTs discovered meeting our criteria. Venkatesh et al described three types of periocular corticosteroid injections with 30 eyes included..$^{14}$ The methods of corticosteroid injection were described as the Smith and Nozik method, the cannula method, and the orbital floor injection method. ${ }^{14}$

There was one RCT performed comparing one dose of intravitreal triamcinolone with one dose of anti-VEGF. ${ }^{57}$ Soheilian et al compared intravitreal triamcinolone with intravitreal bevacizumab with 31 eyes. Intravitreal bevacizumab was randomized to 15 patients and 16 patients received intravitreal triamcinolone. ${ }^{57}$

Tranos et al published an RCT for pars plana vitrectomy compared to standard care in 23 patients (12 randomized to surgery vs eleven randomized to standard of care). Standard care included systemic anti-inflammatory or immunosuppressive agents. ${ }^{58}$

Callanan et al compared an intravitreal fluocinolone acetonide implant of two doses - 0.59 and $2.1 \mathrm{mg}$ - to standard care; however, not all patients had macular edema. The paper mentions a subgroup of 112 eyes having macular edema out of 278 subjects. ${ }^{20}$

Pavesio et al also described the intravitreal fluocinolone acetonide implant for 140 patients with posterior uveitis. Time to recurrence of inflammation of eyes treated with an intravitreal fluocinolone acetone implant $(\mathrm{n}=66)$ was compared to those who received standard care $(n=74)$. Standard care was either systemic prednisolone or combination therapy with an immunosuppressive agent. ${ }^{21}$ Although a subgroup analysis was performed for patients with uveitic macular edema, the number of affected eyes in each group was not documented in the paper. Nor were there any data on the BCVA of these eyes.

Kuppermann et al performed a 6-month study of 315 patients, comparing two separate intravitreal dexamethasone doses compared to observation alone. Each of the three arms in this study had 105 participants. A single injection of the intravitreal dexamethasone was given. Only 27 of these patients had macular edema on subgroup analysis. ${ }^{59}$ Meta-analysis could not be accurately performed for the dexamethasone implant intervention due to trial variation and missing data. 
There is no published RCT for immunomodulatory drugs, including IFNs and anti-TNF- $\alpha$, methotrexate, or macular grid laser.

Acetazolamide was the only intervention with three RCTs. There were two studies from the US ${ }^{60,61}$ and one study from Iran/UK. ${ }^{62}$ A total of 137 eyes were analyzed in the systematic review. The number of eye enrollments in the trials ranged from 30 to $67 .^{60-62}$ Participants were male and female adults.

None of the acetazolamide studies included patients with other ocular conditions affecting assessment and progression of visual acuity, such as central retinal vein occlusion or recent cataract surgery. All trials included patients with clinically significant macular edema. All trials specified uveitic macular edema. ${ }^{60-62}$

All of the trials explicitly report the primary outcome factor of BCVA. ${ }^{60-62}$ Central macula thickness was reported in two of the studies, either at baseline to follow up measurements or using mean change in thickness $(\mu \mathrm{m})$. Duration of uveitis and baseline intraocular pressure measurements were included in the paper by Whitcup et al, ${ }^{60}$ with a mean duration of uveitis in patients of 6.6 years. Only two patients had uveitis for less than 1 year. The duration of uveitis in Lashay et $\mathrm{al}^{62}$ was between 1.5 and 25 years, with a mean duration of 4.6 years.

\section{Acetazolamide Systematic review}

The RCT by Lashay et al was a double-blinded, crossover trial evaluating the effect of acetazolamide on CME in patients with Behçet's disease. ${ }^{62}$ Both patients and investigators were masked for randomization, except one ophthalmologist who monitored patients' compliance and drug-adverse reactions. In the study, 67 eyes of 35 patients were randomized. Study duration was a 42-month period (1996-2000). A total of six patients were lost to follow-up. The test intervention was acetazolamide $250 \mathrm{mg}$ orally every 12 hours for 4 weeks (course A). The control was a placebo tablet orally every 12 hours for 4 weeks (course B). A multivitamin tablet was manufactured in the same shape as acetazolamide and was used as placebo.

The RCT by Whitcup et al was a randomized, double-blinded, crossover trial of 40 eyes treated with acetazolamide versus placebo for CME in patients with uveitis. Those in the active arm were given oral acetazolamide $500 \mathrm{mg}$ every 12 hours for the first 4 weeks of the study, and the control was a placebo every 12 hours for the first 4 weeks of the study and then crossed over. ${ }^{60}$
The RCT by Farber et al evaluated acetazolamide in patients with macular edema due to chronic iridocyclitis. This was a randomized prospective crossover study. ${ }^{6}$

All three studies were crossover trials. All trials attempted to mask participants and investigators. ${ }^{60-62}$ Allocation concealment was not explicitly described in any of the studies. Patients were randomized, but the randomization process was not explicitly discussed in all three studies. Although attempts were made to mask treatment groups, patients in the acetazolamide group experienced a significant amount of side effects and therefore essentially became unmasked.

\section{Types of outcomes}

All trials used visual acuity charts. ${ }^{60-62}$ Two trials utilized fluorescein angiograms in an attempt to quantify the area of macula thickness. ${ }^{61,62}$ Exact definition of visual acuity varied across the trials. One trial used posterior vitreous penetration ratios using vitreous fluorophotometry as a tool for judging uveitic resolution. ${ }^{60}$

Adverse events were documented in all studies (see Methodological quality). Adverse events related to acetazolamide included paresthesia, nausea, drowsiness, weight loss, chronic fatigue, and cutaneous allergic reaction.

\section{Methodological quality}

All three trials were adequately masked. Attempts were made to design the placebo medication to look like acetazolamide. ${ }^{6-62}$ Investigators were masked to treatment allocation for measurement of BCVA, fundus fluorescein angiogram acquisition, and for measurement of posterior vitreous penetration ratio. Vitreous penetration ratios were assessed using vitreous fluorophotometry. This scan measures light given off by intravenously injected fluorescein that has leaked through the retinal vessels into the vitreous. ${ }^{60}$ It is used to detect the breakdown of the blood-retinal barrier. Vitreous ratios are determined with the aid of software program log log plasma (Coherent Medical, Santa Clara, CA, USA). ${ }^{60}$

Investigators performing the baseline characteristics and slit-lamp examinations were also masked. Study data were collected, interpreted, and analyzed by other masked investigators.

All three trials randomized their study participants. ${ }^{60-62}$ Whitcup et $\mathrm{al}^{60}$ described patients being randomized but did not explicitly discuss method of randomization. Lashay et $\mathrm{al}^{62}$ described how both patients and investigators were masked for randomization, except one ophthalmologist who monitored patients' compliance and drug-adverse reactions. The randomization process has not been explicitly 
mentioned in the paper. In Farber et $a l,{ }^{61}$ both the acetazolamide and the placebo tablets were provided by Storz Ophthalmic Pharmaceuticals, and repackaged and labeled by one of the principal investigators. She was the only person who knew which medication each patient received, and the patients were told to contact her if they experienced medication side effects. All other investigators as well as the patients were masked. The randomization process was not explicitly mentioned in the paper. Allocation concealment was not described in any of the trials.

Intention-to-treat analysis was not performed in any of the three studies. Lashay et $\mathrm{al}^{62}$ did not include six patients in the analysis, for reasons of noncompliance. This was not specifically defined, nor were reasons for noncompliance provided.

In Farber et al's RCT, ${ }^{61}$ seven patients discontinued the treatment. Discontinuation was because of fatigue and body rash in patient 1 , fatigue and muscle cramps in patient 2 , hematuria in patient 3 , drug interaction and diuresis in patient 4 , reaction to the fluorescein dye during the fluorescein angiogram from patient 5 and severe fatigue in patient 6 . Patient 7's visual acuity was better than 20/40, so they were also excluded from the study. Two patients had to have their acetazolamide doses reduced due to drowsiness, weight loss, and chronic fatigue. A third patient had their dose of medication reduced due to a mild cutaneous allergic reaction on the body, and swelling of extremities. Overall adverse events included paresthesia, nausea, drowsiness, weight loss, chronic fatigue, and cutaneous allergic reaction. Mild nausea and pins and needles were noted as well.

In Whitcup et al's RCT, ${ }^{60}$ three patients postrandomization were not eligible in the analysis. One patient developed choroidal neovascular membrane. A second developed severe depression from the medication and dropped out of the study, and the third developed a retinal detachment soon after randomization. A further three patients were excluded from the analysis due to progression of existing depression, nausea, and diarrhea and anxiety, with only a total of 34 patients used in the final analysis to report outcome measures.

Adverse events of interest included paresthesia, nausea, drowsiness, weight loss, chronic fatigue, and cutaneous allergic reaction. Mild nausea and pins and needles were noted as well. Ninety-two percent of patients in the acetazolamide group documented adverse drug reactions, compared to $14 \%$ in the placebo group.

\section{Meta-analysis}

Only two trials (Whitcup et $\mathrm{al}^{60}$ and Farber et $\mathrm{al}^{61}$ ) were finally included in the meta-analysis. Lashay et $\mathrm{a}^{62}$ was not included in the meta-analysis, as BCVA for the combined patients or the individual $t$-tests for the two crossover arms were not provided. From the Lashay et al paper, we were unable to correlate the mean difference or standard means with the $t$-tests in the paper. The mean difference was not given explicitly, and neither was it clear how to calculate this with the correlating $P$-values. Table 1 in Lashay et al, titled "Effect of acetazolamide on VA," gives the individual BCVAs for the two arms of the crossover trial: one arm with 27 eyes and the other with 28 eyes. The results section combines the data with a total of 55 eyes. An independent $t$-test was quoted as showing no significant effect of acetazolamide on BCVA of patients $(P=0.53)$, nor on the time effect $(P=0.64)$ or on the carryover effect $(P=0.45)$. This would correlate to weeks 4 , 8 , and 12 . The mean standard deviation or difference could not be manually calculated. Efforts were made to contact the corresponding author to provide us with explanations and the raw data; however, we are still awaiting a response.

In Farber et $\mathrm{al}^{61}$ and Whitcup et al, ${ }^{60}$ mean standard deviation was calculated manually. As the sample size was small, the confidence intervals would have been calculated using a $t$-distribution. Standard error was calculated from the $P$-values. Standard deviation was then calculated using the formula below:

Standard deviation $=\frac{\text { Standard error of difference in means }}{\sqrt{\left(1 / \mathrm{N}_{\mathrm{E}}+1 / \mathrm{N}_{\mathrm{C}}\right)}}$

The overall mean difference for the meta-analysis was -0.01 , with a $95 \%$ confidence interval of -0.17 to 0.15 (Figure 2). $I^{2}$ was zero, as was $\chi^{2}$; degrees of freedom $=1$, $P=0.97 ; Z$-statistic $=0.11, P=0.91$. This highlights that there is no significant effect of acetazolamide on visual acuity for uveitic macular edema.

\section{Interventions}

\section{Results for all types of interventions}

A summary of trials found for treatment of uveitic macular edema up to October 2011 can be found in Supplementary materials. This includes results, complications of the interventions, and trial follow-up times.

\section{Periocular corticosteroid injections}

Venkatesh et $\mathrm{al}^{14}$ was a prospective RCT comparing three types of periocular corticosteroid injections for macular edema secondary to intermediate uveitis. These types were the subTenon cannula method, Smith and Nozik method, and orbital floor injection. Mean visual acuity was calculated using the mean value of the decimal fraction of Snellen visual acuity 


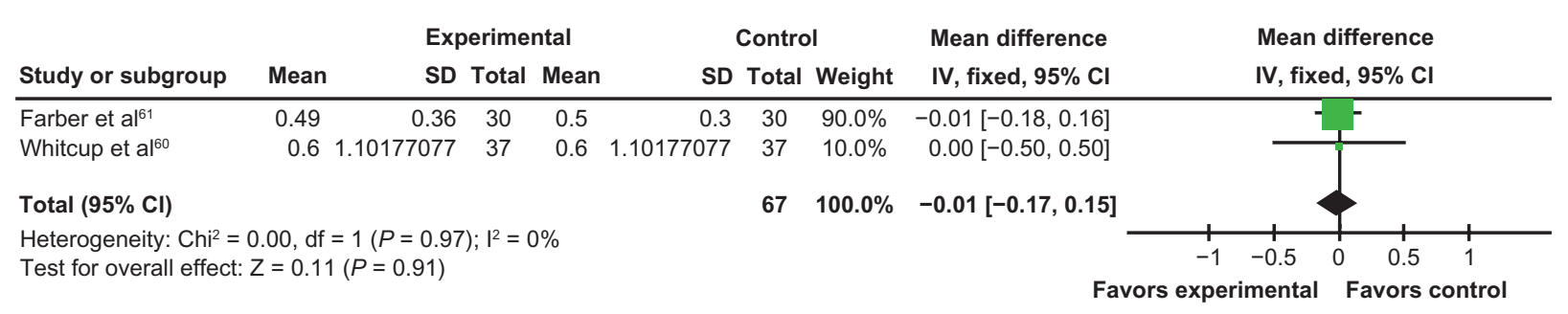

Figure 2 Forest plot.

Abbreviations: SD, standard deviation; IV, intravitreal; $\mathrm{Cl}$, confidence interval.

and logarithm of minimum angle of resolution visual acuity. Mean visual acuity improved significantly in all three groups at 12 weeks $(P=0.00)$, with no statistical difference between the groups. For the Smith and Nozik method, there was an improvement from $0.29 /-0.12$ (mean/standard deviation) to $0.78 /-0.23$; for the cannula method, there was an improvement from $0.25 /-0.08$ to $0.75 /-0.24$; and in the orbital floor method, there was an improvement from $0.24 /-0.10$ to $0.72 /-0.27$. Mean central macular thickness was found to decrease significantly at 12 weeks $(P<0.05)$ : by $32.46 \%$ in the Smith and Nozik group, $43.97 \%$ in the cannula group, and $29.75 \%$ in the orbital floor group. A significant increase in intraocular pressure was reported in eight out of 30 patients in the study. There was no significant difference between the groups in steroid-induced intraocular pressure rise. There was no specific mention of how these patients were treated. Complications, such as ptosis, fat prolapse, and fat necrosis, were not noted. Cataract progression was not documented, perhaps due to the short duration of follow-up (12 weeks). Other case series (Leder et al, ${ }^{63} \mathrm{Jea}$ et al, ${ }^{17}$ and Yoshikawa et $\mathrm{al}^{64}$ ) showed similar results and side-effect profile. Cataract progression was also significant in use of periocular corticosteroids, with studies noting a range of $15.3 \%-17 \%{ }^{14,17,63,64}$

\section{Intravitreal triamcinolone application}

A total of 21 studies were found using IVTA for uveitic macular edema. Most were either clinical studies or case series. There was only one RCT. This was a comparative study with one to three injections of bevacizumab $(1.25 \mathrm{mg})$ and one to three $2 \mathrm{mg}$ injections of IVTA..$^{59}$ Of the 31 eyes in this RCT, 15 eyes were randomized to intravitreal bevacizumab, and 16 eyes received IVTA. There was no significant difference between the two groups in terms of baseline characteristics and no significant difference in results at follow-up. Visual acuity improvement was significant at 36-week follow-up in the IVTA group $(P=0.007)$. Only the IVTA group showed a significant reduction in central macular thickness at 36 weeks $(P=0.049)$. All case series found in our literature search showed either an improvement in mean visual acuity or macular edema. Complication rates were similar, with all documenting an intraocular pressure increase amongst some patients ranging from $22 \%$ to $60 \%$. No subgroup analysis was performed, and since in most studies exclusion criteria included preexisting glaucoma or intraocular pressure above $21 \mathrm{mmHg}$, it is not known whether this treatment can be used in glaucoma patients. ${ }^{63,65-78}$

\section{Corticosteroid implants}

The Jaffe, ${ }^{24}$ Jaffe et al, ${ }^{22,23}$ Kempen et al, ${ }^{25}$ and Lowder et a ${ }^{18}$ papers are worth mentioning, as these RCTs show good results for posterior and chronic uveitis. Macular edema was mentioned in each of these papers, but there was not enough detail to extract specific data in most trials. Kempen et al performed subgroup analysis for macular edema. The proportion of eyes having macular edema in each group was similar at baseline. By 6 months, fewer eyes had macular edema in the implant in the systemic group compared to the implant group (20\% versus $34 \%$ and $40 \%$ versus $48 \%$, respectively; $P<0.001$ ). However, the proportions with macular edema by 24 months did not represent a substantial difference in change from baseline between groups $(P=0.071)$. Callanan et $\mathrm{al}^{20}$ and Pavesio et $\mathrm{a}^{21}$ performed RCTs for the intravitreal fluocinolone acetonide implant, with good long-term follow-up and improvements in visual acuity. The Callanan et al study looked at two different strengths of Retisert implant, whereas Pavesio et al only compared the lower dose. In Callanan et al, the proportion of eyes with reduced $\mathrm{CME}$ was greater in the implanted group compared to the nonimplanted group $(P<0.01)$. There was a reduction in CME in $86 \%$ and $73 \%$ of the implanted eyes compared to $28 \%$ and $28 \%$ of the fellow nonimplanted eyes for the $0.59 \mathrm{mg}$ fluocinolone acetone dose, and CME reduction in $70 \%$ and $45 \%$ of implanted eyes compared to $27 \%$ and $22 \%$ of the fellow nonimplanted eyes for the $2.1 \mathrm{mg}$ fluocinolone acetone dose. In Pavesio et al, both implant and standard care reduced the area of CME. By 2-year follow-up, the proportion of reduced CME was higher in the implant group (86.5\%) compared to standard care $(74.4 \% ; P=0.003)$. 
However, other intravitreal fluocinolone acetonide implant studies showed high complication rates of increased intraocular pressure leading to filtration surgery and cataract progression. ${ }^{22,23}$ Patients requiring ocular antihypertensives ranged from $51 \%$ to $70 \%$. The range of patients requiring filtration surgery in these studies was $5.8 \%-40 \%$. Perhaps more recent studies will have lower rates of filtration surgery due to clinical use of prostaglandin analogs. ${ }^{19,59}$ Kuppermann et al's RCT with implanted dexamethasone in only 27 patients had a better safety profile, with a 10-letter improvement in visual acuity in $54 \%$ of implanted patients compared to $14 \%$ of observed patients. ${ }^{19,59}$ The follow-up, however, was of short duration (6 months). Lowder et al's paper on posterior uveitis ${ }^{18}$ showed good results, and Kuppermann et al's small subgroup analysis specifically for patients with persistent macular edema for greater than 90 days showed promising results. ${ }^{59}$ These studies strengthen the evidence for intravitreal dexamethasone implants to become a real viable intervention for uveitic macular edema. Further studies, specifically for macular edema, of longer duration and good methodological quality are needed.

\section{Acetazolamide and somatostatin analogs}

Three RCTs were conducted for acetazolamide. ${ }^{60-62}$ In Lashay et al, ${ }^{62}$ acetazolamide had no statistically significant effect $(P=0.53)$ on the improvement of visual acuity of patients over that of placebo. In Whitcup et $a 1,{ }^{60}$ there was no statistically significant effect on visual acuity. In Farber et al, ${ }^{61}$ no differences were noted between the acetazolamide and placebo groups. Improved visual acuity was not associated with race or sex. However, younger patients (under age 55 years) were more likely to benefit from treatment. Acetazolamide was associated with adverse outcomes leading to cessation of treatment in certain patients. Paresthesia, nausea, drowsiness, weight loss, chronic fatigue, cutaneous allergic reaction, and depression were amongst the list of complications. Resolution and partial resolution of macular edema and visual acuity were documented, but these studies were not robust in methodological quality. Elevated liver enzymes, pain, and gastrointestinal upset were amongst the complications associated..$^{60-62}$

\section{Anti-VEGF treatment}

Eleven interventional studies were found using anti-VEGF in the treatment of uveitic macular edema. All were of small sample size, except for the safety studies. ${ }^{32,33}$ Case-series size ranged from seven to 34 eyes. ${ }^{79-89}$ One RCT has been performed that compared intravitreal bevacizumab to IVTA. ${ }^{57}$ This RCT showed improvement in BCVA at 12, 24, and 36 weeks compared with baseline values with both interventions. There was no significant difference in the amount of improvement between the two different interventions. However, CMT did not improve in the bevacizumab group, while it did in the IVTA group. This difference was statistically significant. Visual acuity was shown to improve in most of the other nine case series. ${ }^{79-89}$ A range for visual acuities could not be given, as there was variation in how visual acuity data were presented. Trials recorded either percentage improvements, an absolute or mean change from baseline, compared visual acuity to an intervention or standard treatment, or presented just significant changes in visual acuity. Results of each clinical trial are presented in Table S1. Most of the nine studies were statistically significant, but given the poor methodological quality, small sample sizes, and relatively short duration of all the studies, it is uncertain what definitive conclusions can be drawn for this intervention. Complication rates were very low. The endophthalmitis rate was $0.02 \%$, and rhegmatogenous retinal detachment occurred in $0.013 \%$ of eyes. ${ }^{32,33}$ Given the safety profile of anti-VEGF agents, further prospective randomized intervention studies of longer follow-up and larger sample size would be recommended. In particular, this intervention does not carry the intraocular pressure-elevation risk of steroids, and could prove to be a useful second-line alternative should more robust studies yield promising results.

\section{Immunomodulatory drugs, including IFNs and anti-TNF- $\alpha$}

There were nine studies identified that examined different types of immunomodulating drugs for the management of uveitic macular edema. These included IFN- $\alpha-2 \mathrm{~A}$, intravitreal adalimumab, infliximab, mycophenolate, subcutaneous efalizumab, and etanercept. ${ }^{35,37-42,90,91}$ No RCTs have been published, with studies ranging from intervention prospective nonrandomized trials, case series, and retrospective reviews. The nine study results are summarized in Table S1. The most common aim of these studies was to reduce the use of systemic corticosteroids. Systemic side effects were noted with immunomodulatory drugs, such as fatigue, flu-like symptoms, headaches, anorexia, and dizziness. Four studies used IFN- $\alpha$, with two out of 45 patients in Bodaghi et al having major side effects of severe depression and major neutropenia. ${ }^{41}$ Intravitreal adalimumab showed no improvement in macular edema and no side effects. ${ }^{12}$ Other 
studies show improvement in visual acuity to warrant further prospective controlled studies. ${ }^{35-37}$

\section{Intraocular methotrexate}

Only two studies were found for intravitreal methotrexate. ${ }^{51,92}$ One was an interventional case series of 15 patients for uveitic macular edema. ${ }^{92}$ The second paper ${ }^{51}$ looked at intraocular methotrexate in ocular diseases other than primary central nervous system lymphoma, wherein six patients were treated for uveitic macular edema. Both papers showed an improvement in visual acuity and side effects of posterior subcapsular cataract. In Taylor et al, 12 out of 15 patients completed the final follow-up. At 6 months, mean visual acuity improved to 0.59 compared to $1.06(P<0.01) .{ }^{51}$ Macular thickness improved to 275 microns at 6 months compared to baseline $(425, \mathrm{n}=10 ; P<0.01){ }^{92}$

\section{Pars plana vitrectomy}

Six studies reported pars plana vitrectomy as treatment for uveitic macular edema. ${ }^{55,56,58,93-95}$ Gutfleisch et $\mathrm{a}^{55}$ and Sonoda et $\mathrm{al}^{93}$ were prospective nonrandomized interventional studies comparing the intervention with IVTA. Only one study was an RCT. ${ }^{58}$ This consisted of 23 patients randomized into either surgical or medical groups. Mean BCVA in the surgical group improved significantly from $1.0(0.62)$ at baseline to $0.55(0.29)$ at 6 months following vitrectomy $(P=0.011)$, with five $(42 \%)$ eyes reaching vision of $20 / 40$ or better. Mean BCVA in the medical group improved by $0.03(0.27)$ $(P=0.785)$. CME improved in the fluorescein angiogram in four eyes $(33 \%)$, and remained unchanged in seven eyes $(58 \%)$, with increased macular edema in one eye $(8 \%)$. The study was of a short duration, with only 6 months' follow-up. All the studies noted an improvement in BCVA, but five out of six of the studies were interventional case series. Known complications of cataract surgery progression and increased intraocular pressure were documented. ${ }^{55,56,58,93-95}$

\section{Vitamin E}

Nussenblatt et al's RCT showed no effect over an 18-month follow-up for 4 months of 1600 IU of Vitamin $\mathrm{E}$ as an intervention compared to placebo. ${ }^{96}$

\section{Macular grid laser}

In Suttorp-Schulten et al's case series of six eyes where macular grid laser was used as a treatment for uveitic macular edema, BCVA improved significantly for one eye, was stable in three, and deteriorated in two. ${ }^{97}$

\section{Literature overview Summaries of the major reviews conducted on uveitic macular edema}

The complexity of the management of uveitic macular edema has been discussed in the medical literature. Dick acknowledged macular edema to be the predominant cause of visual loss with treatment aimed at suppressing the immune system. He highlighted that successful immune suppression did not always correlate to resolution of macular edema and improved visual acuity. ${ }^{1}$ Okhravi and Lightman's review of the management of CME in 2003 also highlighted the need for aggressive immunosuppression, but acknowledged its limitations when the disease becomes refractory. ${ }^{2}$

Treatment with acetazolamide has been used, but there is little evidence for improved visual acuity in the longer term. Vitrectomy is a treatment option in some patients with persistent edema in the presence of chronic vitreal changes or opacified media with or without vitreoretinal traction at the macula. This invasive procedure is an option for uveitic macular edema refractory to other treatments. ${ }^{1}$

Gulati et $\mathrm{al}^{98}$ and Battaglia-Parodi et $\mathrm{al}^{99}$ reviewed antiVEGF therapy in uveitis but not specifically uveitic macular edema. Both papers highlighted the limitations of evidence from case reports and case series that predominate. Gulati's 2011 paper suggested a possible two-pronged treatment regimen. This focuses on achieving disease quiescence through the use of corticosteroids and/or immunosuppressive agents, while treating complications that arise despite adequate disease quiescence with intravitreal anti-VEGF agents.

Ossewaarde-van Norel and Rothova ${ }^{4}$ and Davis ${ }^{100}$ both highlight weak evidence in the management of uveitic macular edema, with the Davis paper particularly pointing out the need for standardized outcome measures such as Early Treatment Diabetic Retinopathy Study charts and OCT parameters.

Sallam et al $^{101}$ advocate injectable sustained-release corticosteroids for the management of uveitis, but the trials mentioned by Lowder et $\mathrm{al}^{18}$ and Callanan et $\mathrm{al}^{20}$ do not specifically mention macular edema. The paper suggests that implanted dexamethasone delivery systems have a better safety profile than single injection. This was based on Lowder et al's RCT, in which throughout the 26 -week study $23 \%$ of patients in the $0.7 \mathrm{mg}$ implanted group required intraocular pressure-lowering measurements. ${ }^{18}$ Tranos et al's review of all types of macular edema is uncertain of the treatments for uveitic macular edema, stating promise for steroid intraocular 
implants, possibly intravitreal steroids, immunosuppressive agents, and pars plana vitrectomy. ${ }^{39}$

Couch and Bakri ${ }^{15}$ and Cunningham et $\mathrm{al}^{102}$ suggest many of the studies using IVTA control intraocular inflammation, but these effects appeared to be weak or transient. Therefore, reinjection is often required. Jabs et al's guideline from an expert panel reiterates the use of corticosteroids, but there is concern regarding high levels of side effects. ${ }^{12}$ They support the rationale for immunosuppressive drugs, given the complication profile of steroids.

de Smet et al provided an overview of uveitis research that incorporated but was not specific to macular edema. This included a summary of epidemiological research. The paper also commented on epidemiology in relation to age and etiology. Future epidemiological studies were described. ${ }^{5}$ They acknowledged that despite best efforts, there is no way of differentiating treatment-induced disease quiescence from disease remission. Thus, current management for any intervention is of long duration and slow withdrawal. All patients hence need continued observation. The paper highlighted the fact that given traditional medications for uveitis have side effects, future efforts need to focus on toxicity of the interventions as well as efficacy. ${ }^{5}$

Given the medical literature gives us no clear therapy, Dick's final statement holds true in all review papers where treatment options are at present multifaceted and should be tailored for each individual patient. ${ }^{1}$

\section{Discussion}

The successful treatment of uveitic macular edema has been traditionally through immunosuppression with steroid therapy. Different delivery routes for steroids have been innovated to aid efficacy and durability as well as reducing systemic side effects. The diversity of interventions found in the medical literature is evidence of how difficult uveitic macular edema is to treat, especially if it becomes refractory. ${ }^{103}$ Steroid-sparing drugs are showing promise in reducing the side effects of long-term steroid use. Intravitreal methotrexate is a possibility, but with only one specific trial completed to date, more evidence is needed. The same is true for all immunomodulatory drugs, including IFNs and anti-TNF- $\alpha$, as no RCTs have been performed. Anti-VEGF agents have been shown to be safe, but no long-term prospective study has proven them to be effective in uveitic macular edema. Larger clinical trials are also needed. Pars plana vitrectomy is always a last resort; however, this subjects a patient to an invasive procedure with associated intraoperative and postoperative complications.
Steroids have been developed as sustained-released implants. Dexamethasone has shown promise in posterior uveitis and could be a real viable solution for uveitic macular edema. Glaucoma patients with uveitic macular edema miss out on this intervention due to its risks of increased intraocular pressure. All trials conducted excluded these high-risk patients. These implant systems are also invasive and are associated with high rates of filtration surgery. Acetazolamide is not a recommended treatment for uveitic macular edema, with meta-analysis showing no treatment effect compared to placebo. Long-term follow-up studies with considerations for adverse effects need to be quantified and documented to provide a better understanding of risks and benefits for patients with uveitic macular edema.

Case reports and retrospective reviews dominate the medical literature in the treatment of uveitic macular edema, with no RCTs performed for immunomodulatory drugs or anti-VEFG. Other interventions such as steroid implants have only one or two prospective clinical trials, which primarily study uveitic edema with only small subgroup analysis of macular edema patients. The high heterogeneity of this disease is perhaps a limitation for structured comparative studies, and the disease course and recurrence adds to the complexity of treatment.

\section{Conclusion}

More results from RCTs with long follow-up periods are needed for interventions for uveitic macular edema to assist in determining overall long-term benefit of different treatments.

Uveitis is in fact a rare and heterogeneous disease, and as such it is difficult to conduct prospective randomized trials, given challenges with recruitment and achieving adequate sample size.

The only intervention with sufficiently robust RCTs for a meta-analysis was acetazolamide, which was shown to be ineffective in improving vision in eyes with uveitic macular edema, and is clinically now rarely used. Interventions showing promise in this disease include dexamethasone implants, immunomodulatory drugs, and anti-VEGF agents. When macular edema has become refractory after multiple interventions, pars plana vitrectomy could be considered. As the disease pathophysiology is uncertain and the course of disease unpredictable, interventions should be tailored to the individual patient.

\section{Disclosure}

The authors report no conflicts of interest in this work. 


\section{References}

1. Dick A. The treatment of chronic uveitic macular oedema: Is immunosuppression enough? Br J Ophthalmol. 1994;78:1-2.

2. Okhravi N, Lightman S. Cystoid macular edema in uveitis. Ocul Immunol Inflamm. 2003;11:29-38.

3. Karim R, Tang B. Use of antivascular endothelial growth factor for diabetic macular edema. Clin Ophthalmol. 2010;4:493-517.

4. Ossewaarde-van Norel A, Rothova A. Clinical review: Update on treatment of inflammatory macular edema. Ocul Immunol Inflamm. 2011;19:75-83.

5. de Smet M, Taylor S, Bodaghi B, et al. Understanding uveitis: the impact of research on visual outcomes. Prog Retin Eye Res. 2011;30: 452-470.

6. Angunawela RI, Heatley CJ, Williamson TH, et al. Intravitreal triamcinalone acetonide for refractory uveitic cystoid macular oedema: long term management and outcome. Acta Ophthalmol Scand. 2005;83:595-599.

7. Antcliff RJ, Spalton DJ, Stanford MR, Graham EM, Ffytche TJ, Marshall J. Intravitreal triamcinolone for uveitic cystoid macular edema: an optical coherence tomography study. Ophthalmology. 2001; 108:765-772.

8. Choudhry S, Ghosh S. Intravitreal and posterior subtenon triamcinolone acetonide in idiopathic bilateral uveitic macular oedema. Clin Experiment Ophthalmol. 2007;35:713-718.

9. Jonas JB. Intravitreal triamcinolone acetonide for treatment of intraocular oedematous and neovascular diseases. Acta Ophthalmol Scand. 2005;83:645-663.

10. Jonas JB. Intravitreal triamcinolone acetonide: a change in a paradigm. Ophthalmic Res. 2006;38:218-245.

11. Taylor S, Isa H, Joshi L, Lightman S. New developments in corticosteroid therapy for uveitis. Ophthalmologica. 2010;224 Suppl 1:46-53.

12. Jabs DA, Rosenbaum JT, Foster CS, et al. Guidelines for the use of immunosuppressive drugs in patients with ocular inflammatory disorders: recommendations of an expert panel. Am J Ophthalmol. 2000;130:492-513.

13. Tamesis RR, Rodriguez A, Christen WG, Akova YA, Messmer E, Foster CS. Systemic drug toxicity trends in immunosuppressive therapy of immune and inflammatory ocular disease. Ophthalmology. 1996;103:768-775.

14. Venkatesh P, Kumar CS, Abbas Z, Garg S. Comparison of the efficacy and safety of different methods of posterior subtenon injection. Ocul Immunol Inflamm. 2008;16:217-223.

15. Couch SM, Bakri SJ. Intravitreal triamcinolone for intraocular inflammation and associated macular edema. Clin Ophthalmol. 2009;3:41-47.

16. Kooij BV, Rothova A, de Vries P. The pros and cons of intravitreal triamcinolone injections for uveitis and inflammatory cystoid macular edema. Ocul Immunol Inflamm. 2006;14:73-85.

17. Jea SY, Byon IS, Oum BS. Triamcinolone-induced intraocular pressure elevation: intravitreal injection for macular edema and posterior subtenon injection for uveitis. Korean J Ophthalmol. 2006;20:99-103.

18. Lowder C, Belfort R, Lightman S, et al. Dexamethasone intravitreal implant for noninfectious intermediate or posterior uveitis. Arch Ophthalmol. 2011;129:545-553.

19. Kuppermann BD, Blumenkranz MS, Haller JA, et al. Randomized controlled study of an intravitreous dexamethasone drug delivery system in patients with persistent macular edema. Dexamethasone DDS Phase II Study Group. Arch Ophthalmol. 2007;125:309-317.

20. Callanan DG, Jaffe GJ, Martin DF, Pearson PA, Comstock TL. Treatment of posterior uveitis with a fluocinolone acetonide implant: three-year clinical trial results. Arch Ophthalmol. 2008;126:1191-1201.

21. Pavesio C, Zierhut M, Bairi K, Comstock TL, Usner DW. Evaluation of an intravitreal fluocinolone acetonide implant versus standard systemic therapy in non-infectious posterior uveitis. Ophthalmology. 2010;117; $567-575$.

22. Jaffe G, McCallum R, Branchaud R, Skalak C, Butuner Z, Ashton P. Longterm follow-up results of a pilot trial of a fluocinolone acetonide implant to treat posterior uveitis. Ophthalmology. 2005;112: 1192-1198.
23. Jaffe G, Martin D, Callanan D, Pearson A, Levy B, Comstock T. Fluocinolone acetonide implant (Retisert) for noninfectious posterior uveitis: thirty-four-week results of a multicenter randomized clinical study. Ophthalmology. 2006;113:1020-1027.

24. Jaffe G. Reimplantation of a fluocinolone acetonide sustained drug delivery implant for chronic uveitis. Am J Ophthalmol. 2008;145: 667-675.

25. Kempen J, Altaweel M, Holbrook J, et al. Randomized comparison of systemic antiinflammatory therapy versus fluocinolone acetonide implant for intermediate, posterior, and panuveitis: the multicenter uveitis steroid treatment trial. Ophthalmology. 2011;118: 1916-1926.

26. Forrester J, Dick A, McMenamin P, Roberts F. The Eye: Basic Sciences in Practice, 3rd ed. Philadelphia: Saunders; 2008.

27. Kafkala C, Choi JY, Choopong P, Foster CS. Octreotide as a treatment for uveitic cystoid macular edema. Arch Ophthalmol. 2006;124: $1353-1355$.

28. Misotten T, Van Laar JAM, Van der Loos Tl, et al. Octreotide long-acting repeatable for the treatment of chronic macular edema in uveitis. $A m J$ Ophthalmol. 2007;144:838-843.

29. Homsi J, Daud AI. Spectrum of activity and mechanism of action of VEGF/PDGF inhibitors. Cancer Control. 2007;14:285-294.

30. Los M, Roodhart JM, Voest EE. Target practice: lessons from phase III trials with bevacizumab and vatalanib in the treatment of advanced colorectal cancer. Oncologist. 2007;12:443-450.

31. Fine HF, Baffi J, Reed GF, Csaky KG, Nussenblatt RB. Aqueous humor and plasma vascular endothelial growth factor in uveitisassociated cystoid macular edema. Am J Ophthalmol. 2001;132: 794-796.

32. Fintak DR, Shah GK, Blinder KJ, et al. Incidence of endophthalmitis related to intravitreal injection of bevacizumab and ranibizumab. Retina. 2008;28:1395-1399.

33. Ueta T, Yanagi Y, Tamaki Y, Yamaguchi T. Cerebrovascular accidents in ranibizumab. Ophthalmology. 2009;116:362.

34. Meyer CH, Michels S, Rodrigues EB, et al. Incidence of rhegmatogenous retinal detachments after intravitreal antivascular endothelial factor injections. Acta Opthalmol. 2011;89:70-75.

35. Androudi S, Tsironi E, Kalogeropoulos C, Theodoridou A, Brazitikos P. Intravitreal adalimumab for refractory uveitis-related macular edema. Ophthalmology. 2010;117:1612-1616.

36. Tranos P, Wickremasinghe S, Stangos N, Topouzis F, Tsinopoulos I, Pavesio C. Major review: macular edema. Surv Ophthalmol. 2004;49: 470-490.

37. Markomichelakis NN, Theodossiadis PG, Pantelia E, Papaefthimiou S, Theodossiadis GP, Sfikakis PP. Infliximab for chronic cystoid macular edema associated with uveitis. Am J Ophthalmol. 2004;138: 648-650.

38. Wang J, Ibrahim M, Turkcuoglu P, et al. Intercellular adhesion molecule inhibitors as potential therapy for refractory uveitic macular edema. Ocul Immunol Inflamm. 2010;18:395-398.

39. Deuter CM, Kotter I, Gunaydin I, Stubiger N, Doycheva DG, Zierhut M. Efficacy and tolerability of interferon alpha treatment in patients with chronic cystoid macular oedema due to non-infectious uveitis. $\mathrm{Br} J$ Ophthalmol. 2009;93:906-913.

40. Deuter CM, Koetter I, Guenaydin I, Stuebiger N, Zierhut M. Interferon alfa-2a: a new treatment option for long lasting refractory cystoid macular edema in uveitis? A pilot study. Retina. 2006;26: 786-791.

41. Bodaghi B, Gendron G, Wechsler B, et al. Efficacy of interferon alpha in the treatment of refractory and sight threatening uveitis: a retrospective monocentric study of 45 patients. Br J Ophthalmol. 2007;91: 335-339.

42. Paire V, Lebreton O, Weber M. [Effectiveness of interferon alpha in the treatment of uveitis macular edema refractory to corticosteroid and/or immunosuppressive treatment]. $J$ Fr Ophtalmol. 2010;33:152-162. French.

43. Kilmartin DJ, Forrester JV, Dick AD. Rescue therapy with mycophenolate mofetil in refractory uveitis. Lancet. 1998;352:35-36. 
44. Siepmann K, Huber M, Stübiger N, Deuter C, Zierhut M. Mycophenolate mofetil is a highly effective and safe immunosuppressive agent for the treatment of uveitis: a retrospective analysis of 106 patients. Graefes Arch Clin Exp Ophthalmol. 2006;244:788-794.

45. Goldblum R. Therapy of rheumatoid arthritis with mycophenolate mofetil. Clin Exp Rheumatol. 1993;11 Suppl 8:S1117-S1119.

46. Doycheva D, Zierhut M, Blumenstock G, Stuebiger N, Deuter C. Long-term results of therapy with mycophenolate mofetil in chronic non-infectious uveitis. Graefes Arch Clin Exp Ophthalmol. 2011;249: 1235-1243.

47. Enk AH, Knop J. Treatment of pemphigus vulgaris with mycophenolate mofetil. Lancet. 1997;350:494.

48. Daniel E, Thorne JE, Newcomb CW, et al. Mycophenolate mofetil for ocular inflammation. Am J Ophthalmol. 2010;149:423-432.

49. Chang PY, Giuliari GP, Shaikh M, Thakuria P, Makhoul D, Foster CS. Mycophenolate mofetil monotherapy in the management of paediatric uveitis. Eye (Lond). 2011;25:427-435.

50. Muñoz-Fernández S, García-Aparicio AM, Hidalgo MV, et al. Methotrexate: an option for preventing the recurrence of acute anterior uveitis. Eye (Lond). 2009;23:1130-1133.

51. Hardwig PW, Pulido JS, Erie JC, Baratz KH, Buettner H. Intraocular methotrexate in ocular diseases other than primary central nervous system lymphoma. Am J Ophthalmol. 2006;142:883-885.

52. Smith JR, Rosenbaum JT, Wilson DJ, et al. Role of intravitreal methotrexate in the management of primary central nervous system lymphoma with ocular involvement. Ophthalmology. 2002;109:1709-1716.

53. Gangaputra S, Newcomb CW, Liesegang TL, et al. Methotrexate for ocular inflammatory diseases. Ophthalmology. 2009;116:2188-2198.

54. Becker M, Davis J. Vitrectomy in the treatment of uveitis. Am J Ophthalmol. 2005;140:1096-1105.

55. Gutfleisch M, Spital G, Mingels A, Pauleikhoff D, Lommatzsch A, Heiligenhaus A. Pars plana vitrectomy with intravitreal triamcinolone: effect on uveitic cystoid macular oedema and treatment limitations. Br J Ophthalmol. 2007;91:345-348.

56. Dugel PU, Rao NA, Ozler S, Liggett PE, Smith RE. Pars plana vitrectomy for intraocular inflammation-related cystoid macular edema unresponsive to corticosteroids. A preliminary study. Ophthalmol. 1992;99(10):1535-1541.

57. Soheilian M, Rabbanikhah Z, Ramezani A, Kiavash V, Yaseri M, Peyman GA. Intravitreal bevacizumab versus triamcinolone acetonide for refractory uveitic cystoid macular edema: a randomized pilot study. J Ocul Pharmacol Ther. 2010;26:199-206.

58. Tranos P, Scott R, Zambarakji H, Ayliffe W, Pavesio C, Charteris DG. The effect of pars plana vitrectomy on cystoid macular oedema associated with chronic uveitis: a randomized, controlled pilot study. $\mathrm{Br} J$ Ophthalmol. 2006;90:1107-1110.

59. Kuppermann BD, Blumenkranz MS, Haller JA, et al. Randomized controlled study of an intravitreous dexamethasone drug delivery system in patients with persistent macular edema. Arch Ophthalmol. 2007;125:309-317.

60. Whitcup SM, Csaky KG, Podgor MJ, Chew EY, Perry CH, Nussenblatt RB. A randomized, masked, cross-over trial of acetazolamide for cystoid macular edema in patients with uveitis. Ophthalmology. 1996;103:1054-1062.

61. Farber MD, Lam S, Tessler HH, Jennings TJ, Cross A, Rusin MM. Reduction of macular oedema by acetazolamide in patients with chronic iridocyclitis: a randomised prospective crossover study. $\mathrm{Br} \mathrm{J}$ Ophthalmol. 1994;78:4-7.

62. Lashay AR, Rahimi A, Chams H, et al. Evaluation of the effect of acetazolamide on cystoid macular oedema in patients with Behcet's disease. Eye (Lond). 2003;17:762-766.

63. Leder HA, Jabs DA, Galor A, Dunn JP, Thorne JE. Periocular triamcinolone acetonide injections for cystoid macular edema complicating noninfectious uveitis. Am J Ophthalmol. 2011;152:441-448.

64. Yoshikawa K, Ichiishi A, Kotake S, Sasamoto Y, Kosaka S. [Posterior sub-Tenon's injections of repository corticosteroids in uveitis patients with cystoid macular edema]. Nihon Ganka Gakkai Zasshi. 1993;97:1070-1074. Japanese.
65. Teoh SC, Hogan AC, Dick AD, Lee RW. Mycophenolate mofetil for the treatment of uveitis. Am J Ophthalmol. 2008;146:752-760.

66. Young S, Larkin G, Branley M, Lightman S. Safety and efficacy of intravitreal triamcinolone for cystoid macular oedema in uveitis. Clin Experiment Ophthalmol. 2001;29:2-6.

67. Lasave AF, Zeballos DG, El-Haig WM, Diaz-Llopis M, Salom D, Arevalo JF. Short-term results of a single intravitreal bevacizumab (avastin) injection versus a single intravitreal triamcinolone acetonide (kenacort) injection for the management of refractory noninfectious uveitic cystoid macular edema. Ocul Immunol Inflamm. 2009;17: 423-430.

68. Bae JH, Lee CS, Lee SC. Efficacy and safety of intravitreal bevacizumab compared with intravitreal and posterior sub-tenon triamcinolone acetonide for treatment of uveitic cystoid macular edema. Retina. 2011;31: $111-118$.

69. Kok H, Lau C, Maycock N, McCluskey P, Lightman S. Outcome of intravitreal triamcinolone in uveitis. Ophthalmology. 2005;112:1916. e1-e7.

70. Das-Bhaumik RG, Jones NP. Low-dose intraocular triamcinolone injection for intractable macular oedema and inflammation in patients with uveitis. Eye (Lond). 2006;20:934-937.

71. Atmaca LS, Yalcindag FN, Ozdemir O. Intravitreal triamcinolone acetonide in the management of cystoid macular edema in Behçet's disease. Graefes Arch Clin Exp Ophthalmol. 2007;245:451-456.

72. Sallam A, Taylor S, Habot-Wilner Z, et al. Repeat intravitreal triamcinolone acetonide injections in uveitic macular oedema. Letter to the editor. Acta Ophthalmologica. 2011.

73. Karacorlu M, Mudun B, Ozdemir H, Karacorlu SA, Burumcek E. Intravitreal triamcinolone acetonide for the treatment of cystoid macular edema secondary to Behçet disease. Am J Ophthalmol. 2004;138: 289-291.

74. Dong Z, Namba K, Kitaichi N, Goda C, Kitamura M, Ohno S. Efficacy and complications of intravitreal injection of triamcinolone acetonide for refractory cystoid macular edema associated with intraocular inflammation. Jpn J Ophthalmol. 2008;52:374-379.

75. Maca SM, Abela-Formanek C, Kiss CG, Sacu SG, Benesch T, Barisani-Asenbauer T. Intravitreal triamcinolone for persistent cystoid macular oedema in eyes with quiescent uveitis. Clin Experiment Ophthalmol. 2009;37:389-396.

76. Androudi S, Letko E, Meniconi M, Papadaki T, Ahmed M, Foster CS. Safety and efficacy of intravitreal triamcinolone acetonide for uveitic macular edema. Ocul Immunol Inflamm. 2005;13:205-212.

77. Morrison VL, Kozak I, LaBree LD, Azen SP, Kayicioglu OO, Freeman WR. Intravitreal triamcinolone acetonide for the treatment of immune recovery uveitis macular edema. Ophthalmology. 2007;114:334-339.

78. Larsson J, Hvarfner C, Skarin A. Intravitreal triamcinolone in two patients with refractory macular oedema in sarcoid uveitis. Acta Ophthalmol Scand. 2005;83:618-619.

79. Lasave AF, Zeballos DG, El-Haig WM, Diaz-Llopis M, Salom D, Arevalo JF. Short-term results of a single intravitreal bevacizumab (avastin) injection versus a single intravitreal triamcinolone acetonide (kenacort) injection for the management of refractory noninfectious uveitic cystoid macular edema. Ocul Immunol Inflamm. 2009; 17:423-430.

80. Weiss K, Steinbrugger I, Weger M, et al. Intravitreal VEGF levels in uveitis patients and treatment of uveitic macular oedema with intravitreal bevacizumab. Eye (Lond). 2009;23:1812-1818.

81. Mackensen F, Heinz C, Becker MD, Heiligenhaus A. Intravitreal bevacizumab (avastin) as a treatment for refractory macular edema in patients with uveitis: a pilot study. Retina. 2008;28:41-45.

82. Acharya NR, Hong KC, Lee SM. Ranibizumab for refractory uveitisrelated macular edema. Am J Ophthalmol. 2009;148:303-309. e2.

83. Ziemssen F, Deuter CM, Stuebiger N, Zierhut M. Weak transient response of chronic uveitic macular edema to intravitreal bevacizumab (Avastin). Graefes Arch Clin Exp Ophthalmol. 2007;245:917-918.

84. Cordero Coma M, Sobrin L, Onal S, Christen W, Foster CS. Intravitreal bevacizumab for treatment of uveitic macular edema. Ophthalmology. 2007;114:1574-1579. e1. 
85. Mirshahi A, Namavari A, Djalilian A, Moharamzad Y, Chams H. Intravitreal bevacizumab (Avastin) for the treatment of cystoid macular edema in Behçet disease. Ocul Immunol Inflamm. 2009;17:59-64.

86. Fine HF, Baffi J, Reed GF, Csaky KG, Nussenblatt RB. Aqueous humor and plasma vascular endothelial growth factor in uveitis-associated cystoid macular edema. Am J Ophthalmol. 2001;132:794-796.

87. Cervantes-Castañeda RA, Giuliari GP, Gallagher MJ, et al. Intravitreal bevacizumab in refractory uveitic macular edema: one-year follow-up. Eur J Ophthalmol. 2009;19:622-629.

88. Lott MN, Schiffman JC, Davis JL. Bevacizumab in inflammatory eye disease. Am J Ophthalmol. 2009;148:711-717.

89. Al-Dhibi H, Khan AO. Bilateral response following unilateral intravitreal bevacizumab injection in a child with uveitic cystoid macular edema. J AAPOS. 2009; 13:400-402.

90. Neri P, Mariotti C, Cimino L, Mercanti L, Giovannini A. Long-term control of cystoid macular oedema in noninfectious uveitis with mycophenolate mofetil. Int Ophthalmol. 2009;29:127-133.

91. Fukuda K, Kumagai N, Nakamura H, Kubo M, Matsuzaki M, Nishida T. [Successful treatment of cystoid macular edema with etanercept in a patient with rheumatoid arthritis associated panuveitis]. Nihon Ganka Gakkai Zasshi. 2008;112:51-57. Japanese.

92. Taylor SR, Habot-Wilner Z, Pacheco P, Lightman SL. Intraocular methotrexate in the treatment of uveitis and uveitic cystoid macular edema. Ophthalmology. 2009;116:797-801.

93. Sonoda KH, Enaida H, Ueno A, et al. Pars plana vitrectomy assisted by triamcinolone acetonide for refractory uveitis: a case series study. Br J Ophthalmol. 2003;87:1010-1014.

94. Wiechens B, Nölle B, Reichelt JA. Pars-plana vitrectomy in cystoid macular edema associated with intermediate uveitis. Graefes Arch Clin Exp Ophthalmol. 2001;239:474-481.

95. Kiryu J, Kita M, Tanabe T, Yamashiro K, Miyamoto N, Ieki Y. Pars plana vitrectomy for cystoid macular edema secondary to sarcoid uveitis. Ophthalmology. 2001;108:1140-1144.

96. Nussenblatt RB, Kim J, Thompson DJ, et al. Vitamin E in the treatment of uveitis-associated macular edema. Am J Ophthalmol. 2006;141:193-194.

97. Suttorp-Schulten MS, Feron E, Postema F, Kijlstra A, Rothova A. Macular grid laser photocoagulation in uveitis. $\mathrm{Br} J$ Ophthalmol. 1995;79:821-824.

98. Gulati N, Forooghian F, Lieberman R, Jabs D. Vascular endothelial growth factor inhibition in uveitis: a systematic review. Br J Ophthalmol. 2011;95:162-165.

99. Battaglia-Parodi M, Iaconob P, Verbraak FD, Bandelloa F. Antivascular endothelial growth factors for inflammatory chorioretinal disorders. Dev Ophthalmol. 2010;46:84-95.
100. Davis J. Current concepts in the management of uveitic macular edema. Adv Stud Ophthalmol. 2010;7:60-66.

101. Sallam A, Taylor SR, Lightman S. Review and update of intraocular therapy in noninfectious uveitis. Curr Opin Ophthalmol. 2011;22:517-522.

102. Cunningham M, Edelman J, Kaushal S. Intravitreal steroids for macular edema: the past, the present, and the future. Surv Ophthalmol. 2008;53:139-149.

103. Markomichelakis NN, Halkiadakis I, Pantelia E, et al. Patterns of macular edema in patients with uveitis: qualitative and quantitative assessment using optical coherence tomography. Ophthalmology. 2004;111(5):946-953.

104. Roesel M, Gutfleisch M, Heinz C,Heimes B,Zurek-Imhoff B, Heiligenhaus A. Intravitreal and Orbital Floor Triamcinolone Acetonide Injections in Noninfectious Uveitis: A Comparative Study. Ophthalmic Res. 2009;42:81-86.

105. Hogewind BF, Zijlstra C, Klevering BJ, Hoyng CB. Intravitreal triamcinolone for the treatment of refractory macular edema in idiopathic intermediate or posterior uveitis. Eur J Ophthalmol. 2008;18(3):429-434.

106. Smithen LM, Ober MD, Maranan L, Spaide RF. Intravitreal triamcinolone acetonide and intraocular pressure. Am J Ophthalmol. 2004;138(5):740-743.

107. Goldstein DA, Godfrey DG, Hall A, et al. Intraocular pressure in patients with uveitis treated with fluocinolone acetonide implants. Arch Ophthalmol. 2007;125(11):1478-1485.

108. Cox SN, Hay E, Bird AC. Treatment of chronic macular edema with acetazolamide. Arch Ophthalmol. 1988;106(9):1190-1195.

109. Neri P, Mariotti C, Cimino L, Mercanti L, Giovannini A. Long-term control of cystoid macular oedema in noninfectious uveitis with Mycophenolate Mofetil. Int Ophthalmol. 2009;29(3):127-133.

110. Papadaki T,Zacharopoulos I,Iaccheri B,Fiore T Foster CS. Somatostatin for uveitic cystoid macular edema (CME). Ocul Immunol Inflamm. 2005; 13:469-470.

111. Schilling H, Heiligenhaus A, Laube T, Bornfeld N, Jurklies B. Longterm effect of acetazolamide treatment of patients with uveitic chronic cystoid macular edema is limited by persisting inflammation. Retina. 2005;2:182-188.

112. Paire V, Lebreton O, Weber M. [Effectiveness of interferon alpha in the treatment of uveitis macular edema refractory to corticosteroid and or immunosuppressive treatment]. J Fr Ophtalmol. 2010;33:152-162. French.

113. Jabs DA, Nussenblatt RB, Rosenbaum JT. Standardization of Uveitis Nomenclature (SUN) Working Group. Standardization of uveitis nomenclature for reporting clinical data. Results of the First International Workshop. Am J Ophthalmol. 2005;140:509-516. 


\section{Supplementary materials}

\section{Literature search}

\section{Basic Medline search}

1. Macular edema/(3356)

2. Cystoid macular oedema.mp (315)

3. Uveitis/or inflammatory eye disease.mp (8786)

4. Inflammation.mp. or inflammation/(233,027)

5. 3 or $4(240,241)$

6. 1 or $2(3504)$

7. 5 and $6(385)$

8. Find similar to Clinical review: Update on treatment of inflammatory macular edema (9196)

9. Inflammatory macular oedema.mp (1)

10. Inflammatory macular oedema.mp (1)

11. Uveitic macular oedema.mp (10)

12. Find similar to The treatment of chronic uveitic macular oedema (3306)

\section{Detailed Medline search}

1. Randomized controlled trial.pt $(319,877)$

2. (Randomized or randomised).ab,ti $(286,789)$

3. Placebo.ab,ti $(132,213)$

4. Randomly.ab,ti $(163,157)$

5. Groups.ab,ti $(1,094,314)$

6. Dt.fs $(1,504,346)$

7. Trial.ab,ti $(278,821)$

8. 1 or 2 or 3 or 4 or 5 or 6 or $7(2,802,258)$

9. Exp animals $/(15,740,326)$

10. Exp humans/(12,072,823)

11. 9 not $(9$ and 10) $(3,667,503)$

12. 8 not $11(2,377,768)$

13. Exp clinical trial $/(663,572)$

14. (Clin\$ adj3 trial\$).tw $(170,238)$

15. ((Sing1\$ or doubl\$ or trebl\$ or tripl\$) adj3 (blind\$ or mask\$)).tw $(111,244)$

16. Placebo\$.tw $(133,006)$

17. Random $\$ . t w(542,423)$

18. Exp experimental design/(293,871)

19. Exp control group/(1313)

20. 13 or 14 or 15 or 16 or 17 or 18 or $19(1,207,221)$

21. Angiogenes\$.tw. $(45,365)$

22. Exp angiogenesis inhibitors/ $(27,913)$

23. Exp angiogenic factor/(2347)

24. Endothelial cell growth facto\$.tw. (1040)

25. Exp vasculotropin/(27,412)

26. (Macugen\$ or pegaptanib\$ or lucentis\$ or rhufab\$ or ranibizumab\$ or bevacizumab\$).tw (5154)

27. (Anti adj2 VEGF\$).tw (2013)

28. (Endothelial adj2 growth adj2 factor\$).tw $(29,760)$

29. Exp angiogenesis inducing agents/(2347)

30. 21 or 22 or 23 or 24 or 25 or 26 or 27 or 28 or $29(89,768)$
31. Exp macular edema cystoid/(3356)

32. (Macula\$ adj2 edema).tw (4184)

33. CME.tw (2735)

34. CSME.tw (125)

35. (Macula\$ adj2 swell\$).tw (19)

36. Inflammatory macular oedema.tw (1)

37. Uveitic macular oedema.tw (10)

38. 29 or 31 or 32 or 33 or 34 or 35 or 36 or 37 (9999)

39. 20 and 30 and $38(382)$

40. From 39 keep 36, 37, 54, 77, 80, 82, 93-95, 113, 192, $211(12)$

\section{Second Medline search}

1. Macular edema/(3356)

2. Cystoid macular oedema.mp (315)

3. Uveitis/or inflammatory eye disease.mp (8786)

4. Inflammation.mp or inflammation/ $(233,027)$

5. 3 or $4(240,241)$

6. 1 or $2(3504)$

7. 5 and $6(385)$

8. Find similar to Clinical review: Update on treatment of inflammatory macular edema (9196)

9. Inflammatory macular oedema.mp (1)

10. Inflammatory macular oedema.mp (1)

11. Uveitic macular oedema.mp (10)

12. Find similar to The treatment of chronic uveitic macular oedema (3306)

13. Randomized controlled trial.pt $(319,877)$

14. (Randomized or randomised).ab,ti $(286,789)$

15. Placebo.ab,ti $(132,213)$

16. Randomly.ab,ti $(163,157)$

17. Groups.ab,ti $(1,094,314)$

18. Dt.fs $(1,504,346)$

19. Trial.ab,ti $(278,821)$

20. 13 or 14 or 15 or 16 or 17 or 18 or $19(2,802,258)$

21. Exp animals $/(15,740,326)$

22. Exp humans/(12,072,823)

23. 21 not (21 and 22) $(3,667,503)$

24. 20 not $23(2,377,768)$

25. Exp clinical trial $/(663,572)$

26. (Clin\$ adj3 trial\$).tw $(170,238)$

27. ((Singl\$ or doubl\$ or trebl\$ or tripl\$) adj3 (blind\$ or mask\$)).tw $(111,244)$

28. Placebo\$.tw $(133,006)$

29. Random \$.tw $(542,423)$

30. Exp experimental design/(293,871)

31. Exp control group/(1313)

32. 25 or 26 or 27 or 28 or 29 or 30 or $31(1,207,221)$

33. Angiogenes\$.tw $(45,365)$

34. Exp angiogenesis inhibitors/(27,913)

35. Exp angiogenic factor/(2347) 
36. Endothelial cell growth facto\$.tw (1040)

37. Exp vasculotropin/(27,412)

38. (Macugen $\$$ or pegaptanib\$ or lucentis $\$$ or rhufab\$ or ranibizumab\$ or bevacizumab\$).tw (5154)

39. (Anti adj2 VEGF\$).tw (2013)

40. (Endothelial adj2 growth adj2 factor\$).tw $(29,760)$

41. Exp angiogenesis inducing agents/(2347)

42. 33 or 34 or 35 or 36 or 37 or 38 or 39 or 40 or 41 $(89,768)$

43. Exp macular edema cystoid/(3356)

44. (Macula\$ adj2 edema).tw (4184)

45. CME.tw (2735)

46. CSME.tw (125)

47. (Macula $\$$ adj2 swell\$).tw (19)

48. Inflammatory macular oedema.tw (1)

49. Uveitic macular oedema.tw (10)

50. 41 or 43 or 44 or 45 or 46 or 47 or 48 or 49 (9999)

51. 32 and 42 and 50 (382)

52. From 51 keep 36, 37, 54, 77, 80, 82, 93-95, 113, 192, $211(12)$

53. Find similar to Intravitreal bevacizumab versus triamcinolone acetonide for refractory uveitic cystoid macular edema: a randomized pilot study (36)

54. Uveitic macular oedema.tw (10)

55. Exp macular edema cystoid/(3356)

56. Uveitis, anterior/or uveitis, suppurative/or uveitis/or uveitis, posterior/or uveitis, intermediate/or uveitis.mp $(14,973)$

57. Inflammatory.mp $(387,095)$

58. 56 or $57(399,164)$

59. 55 and $58(602)$

60. 55 and $56(351)$

61. From 52 keep 1-12 (12)

62. From 54 keep 1-8 (8)

63. From 60 keep $2,4,8,11,16,20,23,24,28,30,32,35$, $36,38,41,43,46,48,52,55,58,59,61-64,67,68,70$, $73,86,88,92,95,96,98,101-106,111-113,116,119$, $122,125,128-133,137,141,143-147,155,157,161$, $165,166,175,181,182,187,207,209,213,224,231$ (76)

64. From 60 keep 272-274, 277, 278, 286, 302-304 (9)

65. 52 or 54 or 61 or 62 or 63 or 64 (101)

\section{Embase literature search}

1. Randomized controlled trial.pt

2. (Randomized or randomised).ab,ti

3. Placebo.ab,ti

4. Randomly.ab,ti

5. Groups.ab,ti

6. Dt.fs

7. Trial.ab,ti
8. 1 or 2 or 3 or 4 or 5 or 6 or 7

9. Exp animals/

10. Exp humans/

11. 9 not (9 and 10)

12. 8 not 11

13. Exp clinical trial/

14. (Clin $\$$ adj3 trial\$).tw

15. ((Singl\$ or doubl\$ or trebl\$ or tripl\$) adj3 (blind\$ or mask\$)).tw

16. Placebo\$.tw

17. Random\$.tw

18. Exp experimental design/

19. Exp control group/

20. 13 or 14 or 15 or 16 or 17 or 18 or 19

21. Angiogenes $\$ . t w$

22. Exp angiogenesis inhibitors/

23. Exp angiogenic factor/

24. Endothelial cell growth facto\$.tw

25. Exp vasculotropin/

26. (Macugen $\$$ or pegaptanib\$ or lucentis $\$$ or rhufab\$ or ranibizumab\$ or bevacizumab\$).tw

27. (Anti adj2 VEGF\$).tw

28. (Endothelial adj2 growth adj2 factor\$).tw

29. Exp angiogenesis inducing agents/

30. 21 or 22 or 23 or 24 or 25 or 26 or 27 or 28 or 29

31. Exp macular edema cystoid/

32. (Macula $\$$ adj2 edema).tw

33. CME.tw

34. CSME.tw

35. (Macula $\$$ adj2 swell\$).tw

36. Inflammatory macular oedema.tw

37. Uveitic macular oedema.tw

38. 29 or 31 or 32 or 33 or 34 or 35 or 36 or 37

39. 20 and 30 and 38

40. From 39 keep 36, 37, 54, 77, 80, 82, 93-95

41. Find similar to Intravitreal bevacizumab versus triamcinolone acetonide for refractory uveitic cystoid macular edema: a randomized pilot study

42. Uveitic macular oedema.tw

43. Exp macular edema cystoid/

44. Uveitis, anterior/or uveitis, suppurative/or uveitis/or uveitis, posterior/or uveitis, intermediate/or uveitis.mp

45. Inflammatory.mp

46. 44 or 45

47. 43 and 46

48. 43 and 44

49. From 40 keep $1-12$

50. From 42 keep $1-8$

51. From 48 keep 2, 4, 8, 11, 16, 20

76 clinical studies found. 


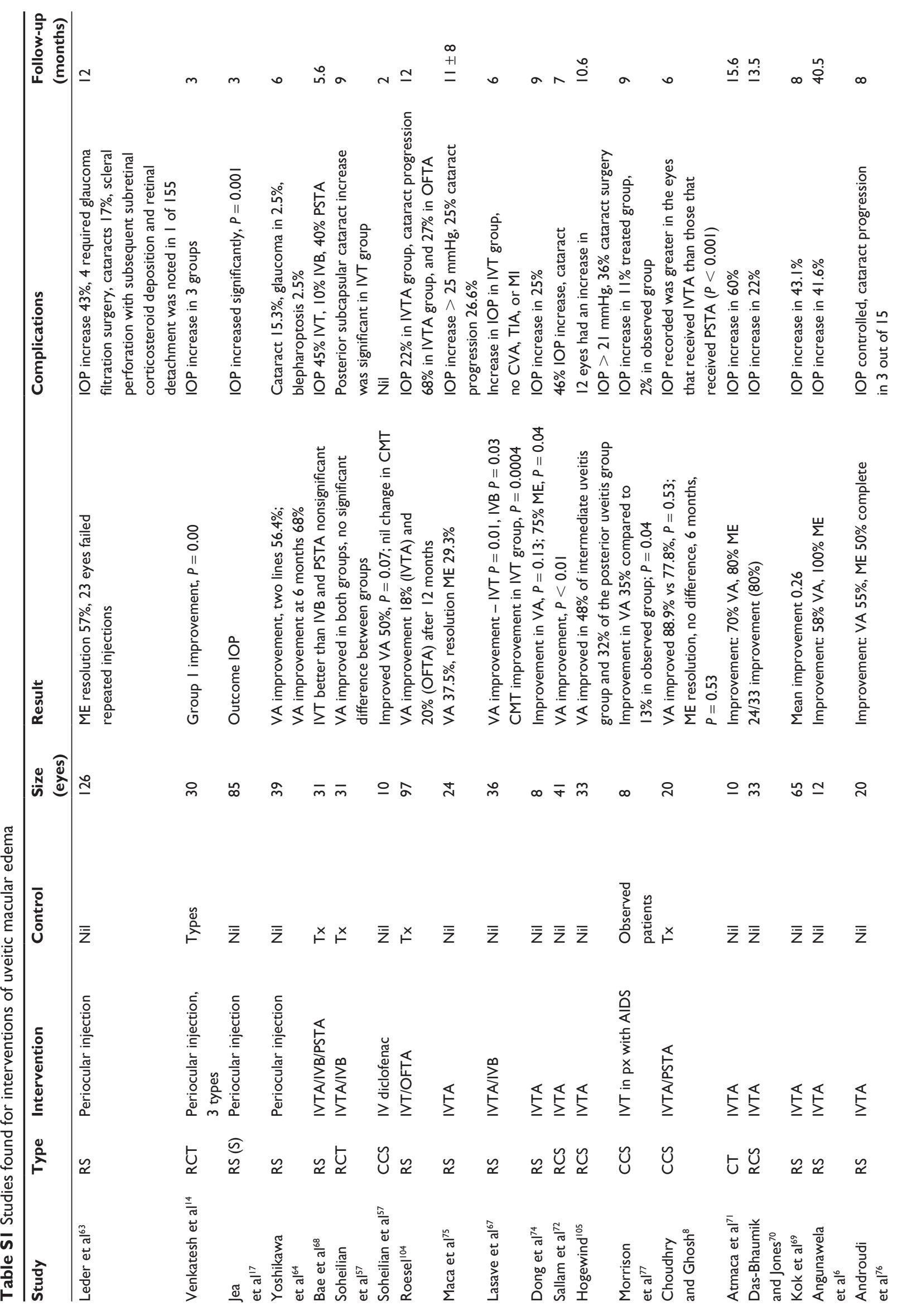




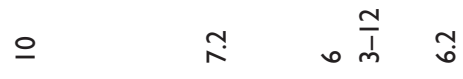

ป

$\stackrel{m}{m}$

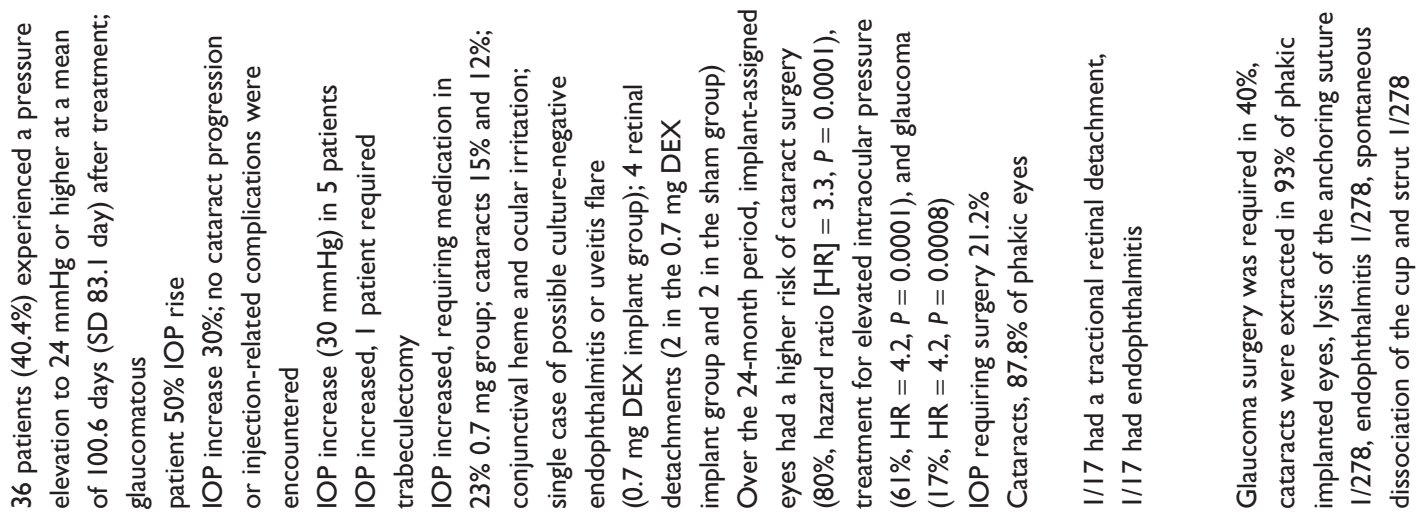
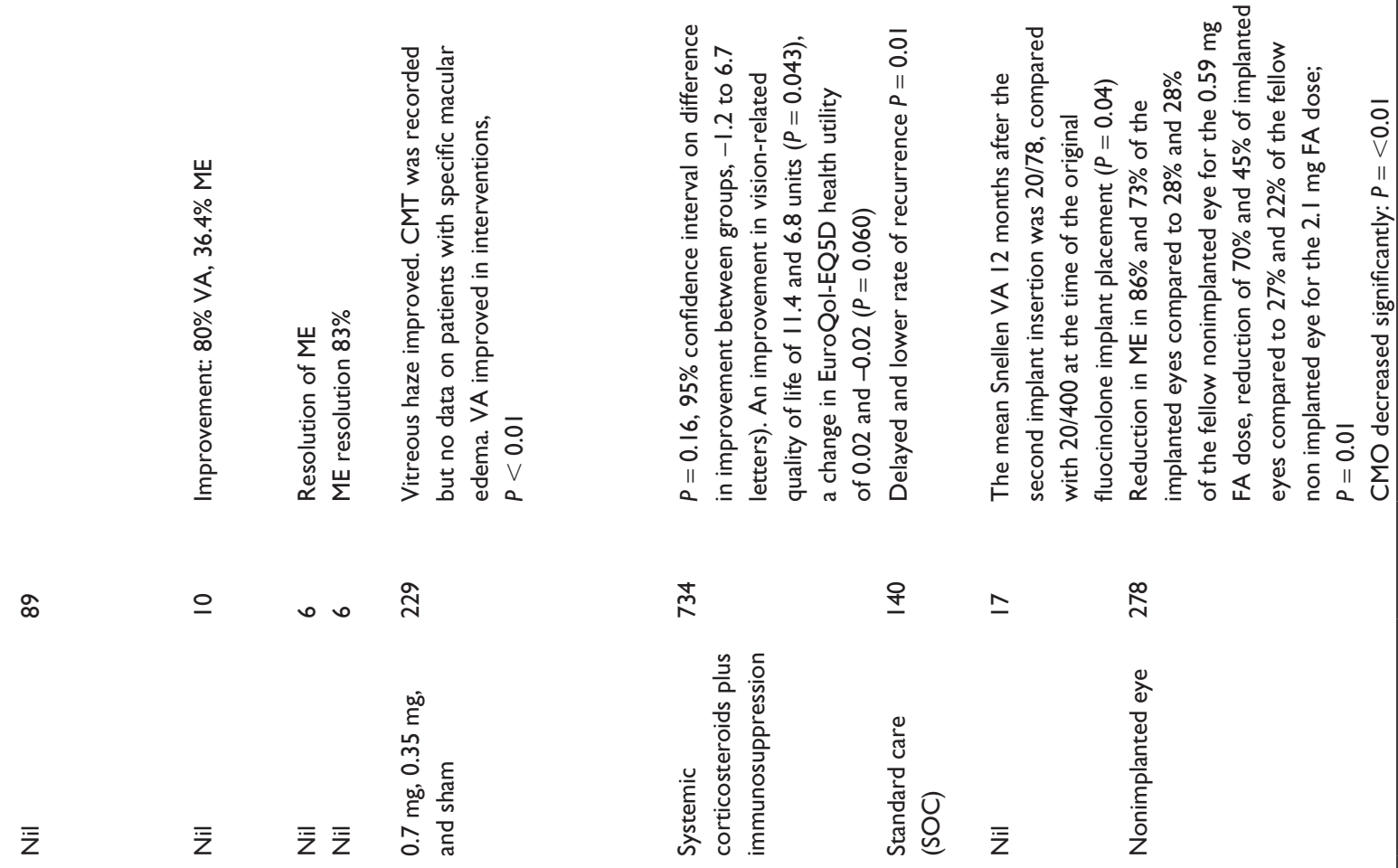

$\overline{\bar{z}}$.

के

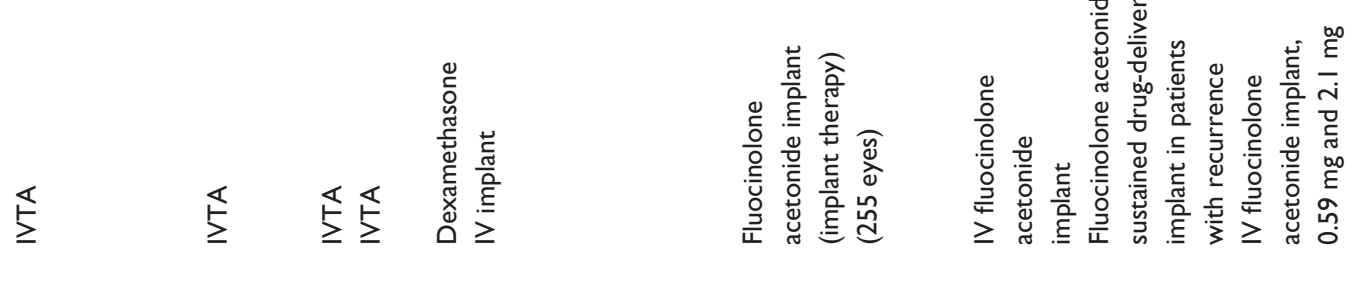

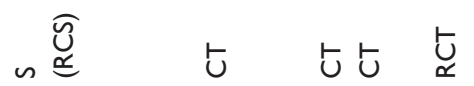

$\begin{array}{llll}\longleftarrow & \longleftarrow & \ddots & \longleftarrow\end{array}$

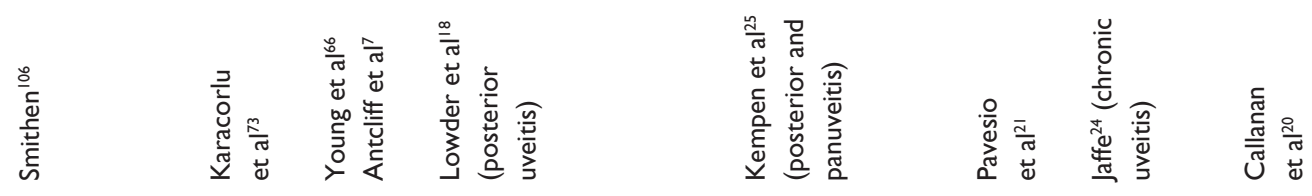




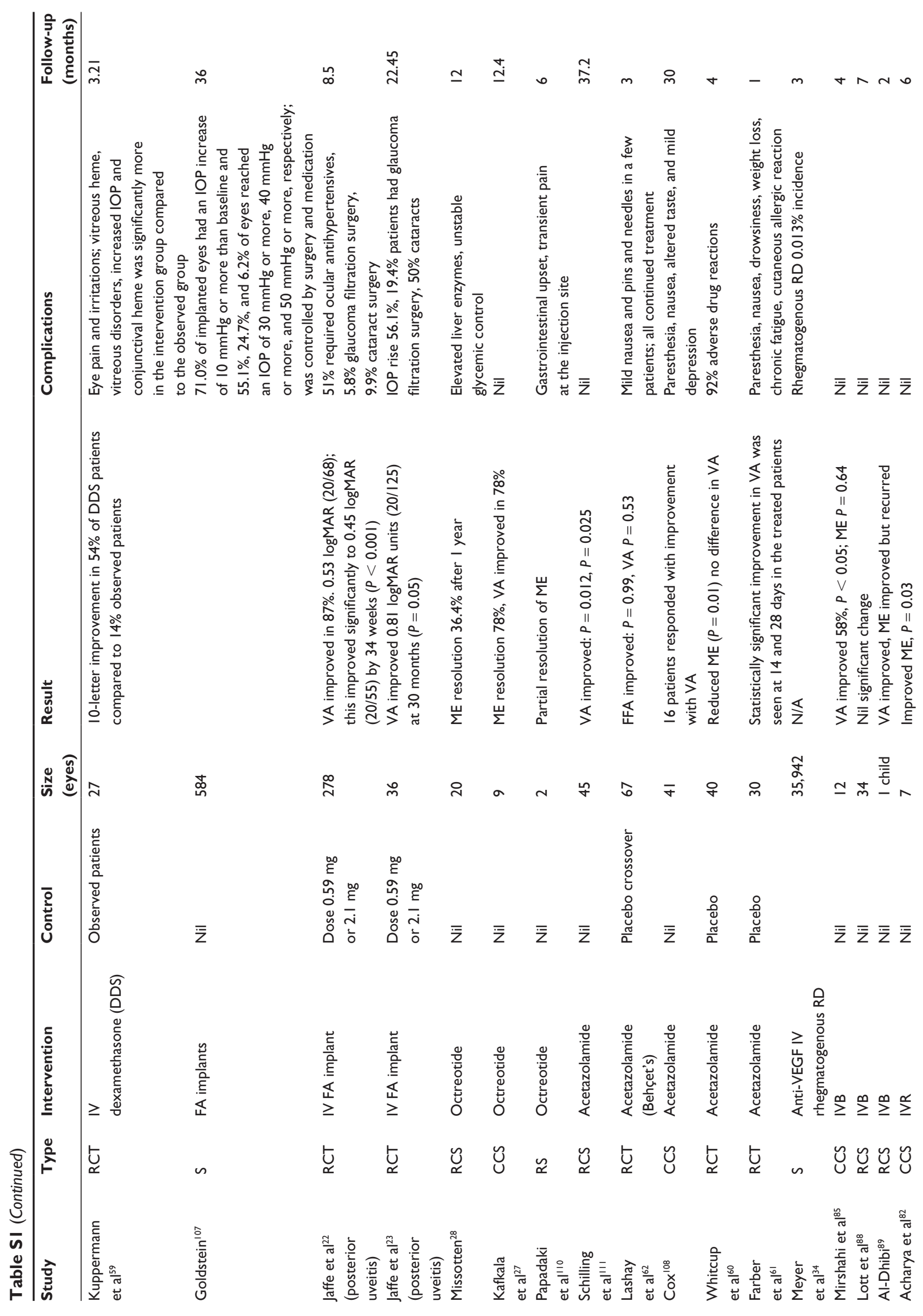



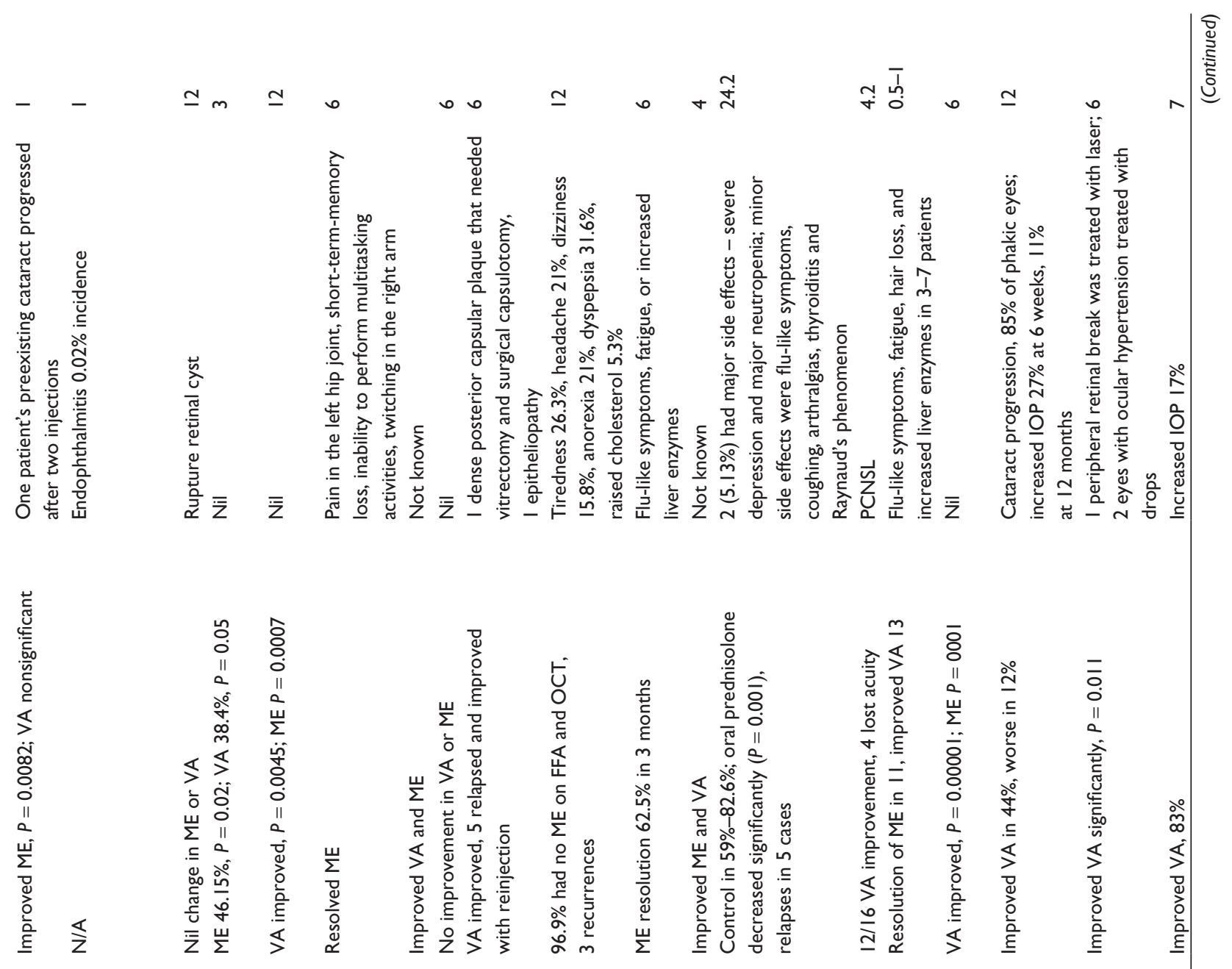

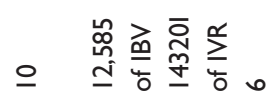

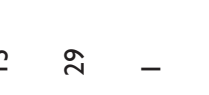

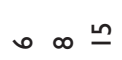

$\frac{4}{2} \sim \frac{2}{2}$

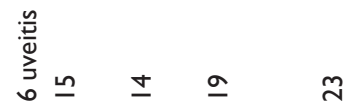

$\overline{\bar{z}} \quad \begin{array}{lllllll}\mathbf{z} & \bar{z} \overline{\bar{z}} & \overline{\bar{z}} & \overline{\bar{z}} & \overline{\bar{z}} \overline{\bar{z}} \overline{\bar{z}} & \overline{\bar{z}}\end{array}$

$\overline{\bar{z}} \bar{z}$

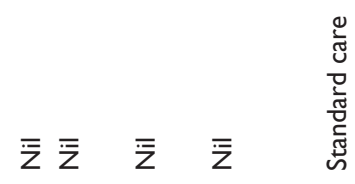

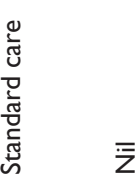

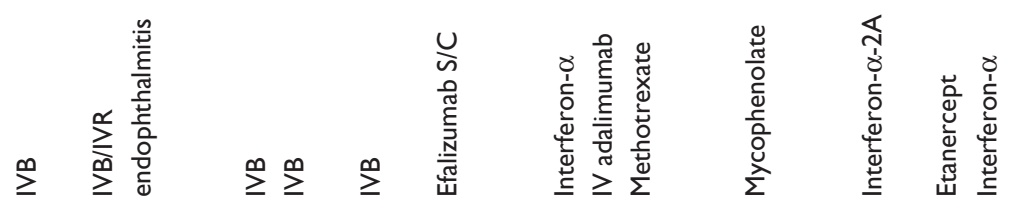

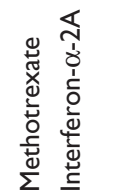

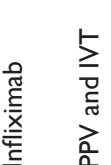

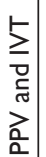

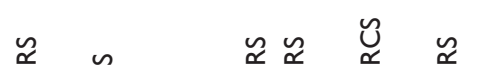

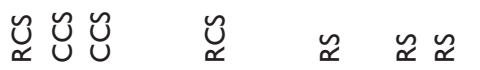

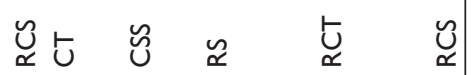

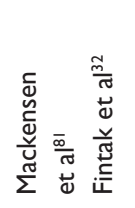

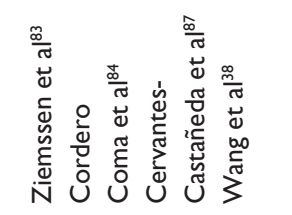

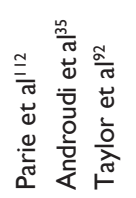

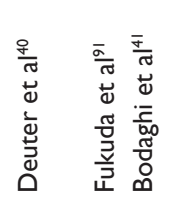

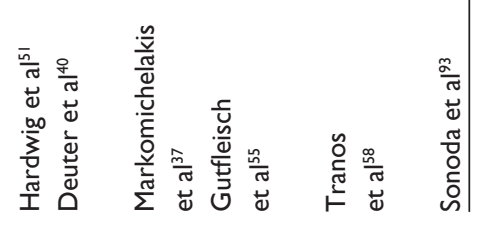




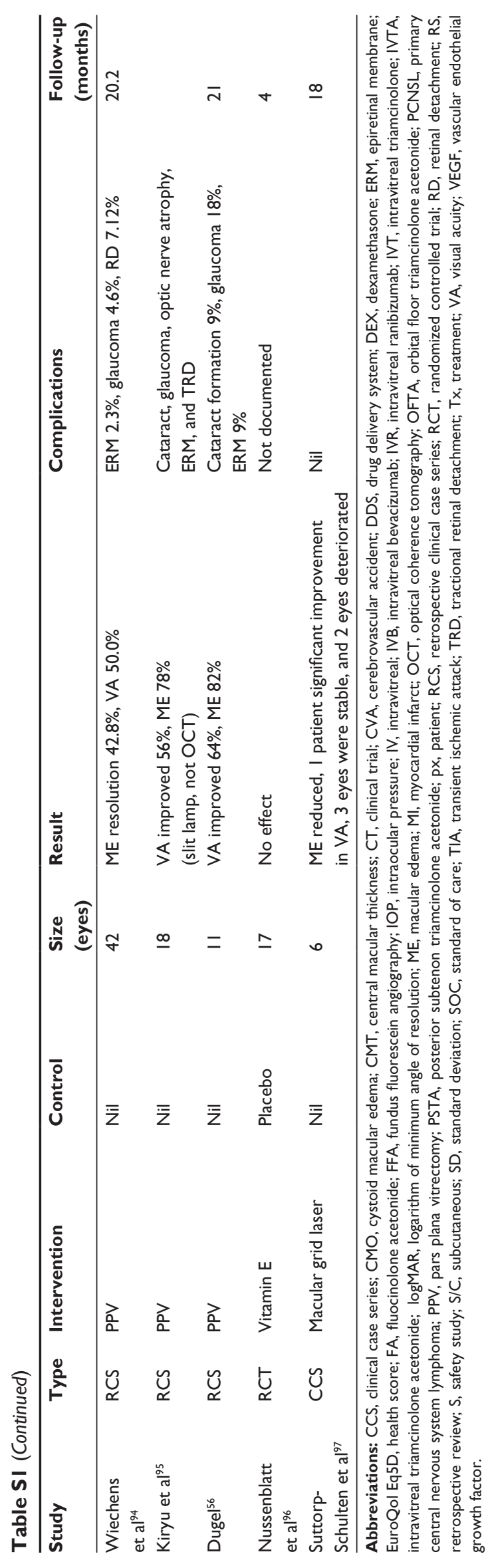




\section{Characteristics of ongoing studies}

Shree Kurup

Title: Evaluation of the utility of intravitreal vascular endothelial growth factor (VEGF) blockade with pegaptanib in cystoid macular edema (CME) associated with non infectious intermediate and panuveitis in an open-label, nonrandomized, uncontrolled interventional pilot trial.

\section{Methods}

Study design: open-label, nonrandomized, uncontrolled interventional pilot trial

\section{Method of randomization: N/A}

Number of participants: 5 patients (laterality not mentioned), finished recruiting

\section{Method of allocation concealment: N/A}

Outcome assessor masking: open-label

Study duration: aiming for 36 months

Losses to follow-up: not known

Intention-to-treat analysis: unknown

\section{Participants}

Inclusion criteria:

- Male and female adults ( $>18$ years of age) with noninfectious uveitis

- Demonstrable (fluorescein angiography [FA] and/or optical coherence tomography [OCT]) bilateral or unilateral CME associated with uveitis of greater than 3 months' but less than 1 year's duration that is documented by two independent qualified observers

- Best-corrected visual acuity (BCVA) between 20/40 and 20/200, as measured by the Early Treatment of Diabetic Retinopathy Study (ETDRS) chart, attributable to CME in the study eye

- Patients may be receiving systemic therapy for the treatment of their intraocular inflammation or CME, or may have been treated for the CME in the past

- Anterior chamber inflammation equal to or greater than 1 and vitreous inflammation equal to or greater than 1 cell and 1 haze, as per the Standardization of Uveitis working group definition ${ }^{113}$
- Females of childbearing potential must agree to utilize effective contraception during the study and 2 months after the last dose of study medication

- Male study patients will agree to use effective contraception

- Ability to give informed consent.

Exclusion criteria:

- Allergy to pegaptanib or any of its components

- Diabetic retinopathy, macular degeneration, or any other ocular condition affecting the study eye that may cause vision loss or in the opinion of the study investigator would interfere with the evaluation of the efficacy of Macugen for the treatment of uveitis-associated CME

- Refusal to try the therapeutic alternative pegaptanib

- Lack of understanding of the consent or protocol

- Suspicion/proved history or current diagnosis (clinical or otherwise) of infectious uveitis

- Need for intraocular surgery within 30 weeks of study duration

- Periocular steroids to the study eye less than 6 weeks prior to study enrollment

- History of any prior intravitreal injections in study eye

- Systemic immunomodulatory agent(s) added or increased in dosage ( $>20 \%$ ) within the last 2 months prior to study enrollment, or potential need for any increase during the study

- Requirement for systemic corticosteroids in the equivalent of oral prednisone $>30 \mathrm{mg} /$ day

- Topical prostaglandin analog use

- Severe debilitating disease or medical problems that make consistent follow-up over the treatment period unlikely (eg, liver impairment, stroke, severe myocardial infarction, terminal cancer)

- History of hypersensitivity to fluorescein or multiple drug allergies that may increase the chance of a drug reaction to Macugen

- Unclear media that precludes assessment of CME in eligible eye(s), such as a cataract or vitreal opacity

- Evidence of a macular hole in the study eye

- Prior or current retinal detachment in the study eye

- Concurrent treatment with any new investigational drug

- Pregnant or lactating women (pregnant and lactating women are excluded, since pregnancy may have some effect on CME)

- Inability to comply with the study requirements 
Type of ME: uveitic CME

Age: not known

Comparability of baseline characteristics: not known

Interventions: pegaptanib (Macugen)

Test intervention: intravitreal (IV) pegaptanib $0.3 \mathrm{mg}$ every 6 weeks as needed for a total of no more than 5

Control: none

\section{Outcomes}

Primary outcome: VA improvement

Measurement of primary outcome: improvement in visual acuity (VA) ETDRS $\geq 15$ letters (time frame: 32 weeks)

Measurement of secondary outcome:

- Proportion of patients experiencing $>0$-letter vision gain and $<15$ loss

- Decrease in CME as evidenced by imaging (FA and 50-micron change in OCT)

- A change in anterior chamber cells or vitreous cells or haze in injected eye

- Change in immunomodulatory medications (topical, periocular, or systemic) after the initiation of Macugen therapy

Results: N/A

Funding source: not known

Country: USA

\section{Thomas A Albini}

Title: Pilot study of ranibizumab (Lucentis) for uveitic $\mathrm{CME}$

\section{Methods}

Study design: intervention model single-group assignment open-label study

Method of randomization: N/A

Number of participants: 10 (estimated)

Method of allocation concealment: N/A
Outcome assessor masking: open-label

Study duration: 24 months (anticipated completion date was July 2010)

Losses to follow-up: N/A

Intention-to-treat analysis: N/A

\section{Participants}

Inclusion criteria:

- Ability to provide written informed consent and comply with study assessments for the full duration of the study

- Age $>18$ years

- Noninfectious uveitis in study eye

- Stable antiuveitis medical regimen for at least 1 month prior to injection and controlled uveitis in the judgment of the investigator

- Vision 20/40 or worse in study eye

- CME on FA

- OCT demonstrating thickness greater than 300 microns in the central subfield

- Media clarity, pupillary dilation, and patient cooperation sufficient to allow OCT testing and retinal photography

Exclusion criteria:

- Previous intravitreal triamcinolone injection in study eye within 3 months of study injection

- Use of more than two glaucoma medicines for study eye

- Significant epiretinal membrane, as judged by treating physician

- Evidence of vitreomacular traction on OCT

- Previous vitrectomy in study eye

- Pregnancy (positive pregnancy test) or lactation

- Premenopausal women not using adequate contraception. The following are considered effective means of contraception: surgical sterilization or use of oral contraceptives, barrier contraception with either a condom or diaphragm in conjunction with spermicidal gel, an intrauterine device, or contraceptive hormone implant or patch

- Any other condition that the investigator believes would pose a significant hazard to the subject if the investigational therapy were initiated

- Participation in another simultaneous independent trial 
- Treatment for CME with intravitreal Lucentis, Macugen, or Avastin within 6 weeks prior to enrollment in this study

- Uncontrolled inflammation in the study eye

- Current vitreous hemorrhage

- Active infectious conjunctivitis, keratitis, scleritis, or endophthalmitis in either eye

- Known allergy to any component of the study drug

- Intraocular pressure $>25 \mathrm{mmHg}$ despite treatment with glaucoma medications

- Blood pressure $>180 / 110$ (systolic above 180 or diastolic above 110). If blood pressure is brought below 180/110 by antihypertensive treatment, the subject can become eligible

- Major nonocular surgery planned during the next 6 months

- Any other condition that the investigator believes would pose a significant hazard to the subject if the investigational therapy were initiated

- No Avastin use permitted in fellow eye during study

- Unwilling or unable to follow or comply with all studyrelated procedures

Type of ME: uveitic CME

Age: unknown

Comparability of baseline characteristics: unknown

Interventions: ranibizumab (Lucentis)

Test intervention: intravitreally administered $0.5 \mathrm{mg}$ ranibizumab

Control: N/A

\section{Outcomes}

Primary outcome: Determine whether patients who receive intravitreal ranibizumab according to the study schedule have improved vision as measured by ETDRS at 4 meters

Measurement of primary outcome: as above

Secondary outcome:

- The mean change in best-corrected visual acuity (BCVA) (assessed by the ETDRS chart at 4 meters) from baseline at 12 months will be computed with a $t$-test (time frame: 1 year)
- The percentage of patients with 15 letters (3 lines) of VA improvement at 30, 60, 90, 120 days, and 12 months (time frame: 1 year)

- The mean change in foveal retinal thickness from baseline at 7 days, and at 30, 60, 90, 120 days, and 12 months will be computed using a $t$-test (time frame: 1 year)

- The incidence of ocular and nonocular adverse events will be evaluated through month 24 (time frame: 2 years)

Measurement of secondary outcome: as above

Results: N/A

Funding source: unknown

Country: USA

\section{Ursula Schmidt-Erfurth}

Title: Functional and morphologic aspects of intravitreal triamcinolone for uveitis-associated CME

\section{Methods}

Study design: prospective observational

Method of randomization: N/A

Number of participants: 30

Method of allocation concealment: N/A

Outcome assessor masking: N/A

Study duration: 24 months

Losses to follow-up: unknown

Intention-to-treat analysis: N/A

Participants

Inclusion criteria:

- Intraocular inflammation

- Able to read

- 18 years old

Exclusion criteria:

- Cataract

- Amblyopia

- Dyslexia

- Already applied intravitreal triamcinolone for current CME 
Type of ME: uveitic CME

Age: unknown

Comparability of baseline characteristics: unknown

Interventions: intravitreal triamcinolone acetonide (IVTA)

Test intervention: IVTA

Control: N/A

\section{Outcomes}

Primary outcome: retinal anatomy was evaluated using OCT

Measurement of primary outcome: Cirrus HD-OCT

Secondary outcome: VA, contrast sensitivity, funduscontrolled microperimetry

Measurement of secondary outcome: visual function testing consisted of assessing ETDRS distance VA, reading acuity and reading speed using a standardized German-language test (Radner reading charts), contrast sensitivity using PelliRobson contrast sensitivity charts, and fundus-controlled microperimetry using the MP-1 Microperimeter (Nidek)

Results: unknown

Funding source: N/A

Country: Austria

\section{No corresponding author}

Title: Vitamin E to treat uveitis-associated macular edema

\section{Methods}

Study design: double-masked, randomized study

Method of randomization: unknown

Number of participants: 80 (estimated enrollment)

Method of allocation concealment: N/A

Outcome assessor masking: unknown

Study duration: 72 months
Losses to follow-up: unknown

Intention-to-treat analysis: unknown

\section{Participants}

Inclusion criteria:

- Patients with documented CME associated with intraocular inflammatory disease. This should be documented both by clinical examination and by FA and should be agreed upon by two independent observers

- Patients must have at least one eye with BCVA of 20/32 or less (as measured by the ETDRS chart) and ME. These will be considered eligible eyes.

- A patient must have at lease one eligible eye with clinical opacity grades of less than or equal to 1 for posterior subcapsular opacity and less than or equal to 2 for nuclear opacity

- Patients 9 years and above, of either sex (nonpregnant females), who carry the diagnosis of endogenous anterior, intermediate, posterior, or panuveitis

- Patients may be receiving systemic therapy for the treatment of their CME, or may have been treated for the CME in the past

- Vitreous haze in both eyes equal to or less than 1 cell and 1 haze

- The patient or patient's guardian must understand and sign the protocol informed consent and/or assent document

Exclusion criteria:

- Vitamin E supplementation over and above the amount in a multivitamin (60 IU/day) 1 month prior to entry into the study

- History of hypersensitivity to fluorescein

- Unclear media that precludes assessment of CME in eligible eye(s), such as a cataract or vitreal opacity

- Evidence of a macular subretinal neovascular net or a macular hole in the eligible eye(s)

- Prior or current macular detachment in eligible eye(s)

- Concurrent Coumadin (warfarin) therapy or known bleeding diathesis

- Concurrent treatment with a new investigational drug

- Malabsorption syndrome

- Concurrent administration of anticholesterol resin medications (eg, cholestyramine)

- Concurrent administration of the antiobesity drug orlistat

- Pregnant or lactating women

- Medical problems that make consistent follow-up over the treatment period unlikely (eg, stroke, severe myocardial infarction, terminal carcinoma) 
- Inability to comply with the study requirements

- Severe optic nerve atrophy in eligible eye(s)

- History of intracranial bleeds

Type of ME: uveitic CME

Age: unknown

Comparability of baseline characteristics: unknown

Interventions: Vitamin E

Test intervention: $1600 \mathrm{IU} /$ day of Vitamin E for 4 months

Control: placebo

\section{Outcomes}

Primary outcome: VA increase of ten letters or more from baseline to month 4

Secondary outcome: total area of leakage and macular height as determined by FA, changes in CME as measured by stereoscopic color photographs, OCT, the need for periocular injections or additional systemic immunomodulatory medications

Measurement of secondary outcome: FA, OCT

Results: unknown

Funding source: unknown

Country: USA

\section{Masoud Soheilian (I)}

Title: The comparison between the therapeutic effect of intravitreal diclofenac and triamcinolone in persistent uveitic cystoids macular edema

Methods

Study design: randomized interventional parallel assignment, double-blinded

Method of randomization: unknown

Number of participants: unknown

Method of allocation concealment: unknown

Outcome assessor masking: yes
Study duration: unknown

Losses to follow-up: unknown

Intention-to-treat analysis: unknown

Participants

Inclusion criteria:

- CME diagnosed by OCT and fluorescein angiography

- $5 / 200<\mathrm{VA}<20 / 50$

- Resistance to routine treatment (oral treatment, periocular injection)

Exclusion criteria:

- History of retinal disease, eg, diabetes, causing ME

- Arterial occlusion

- Mono-ocular patients

- Candidates for intraocular operation

- History of glaucoma or ocular hypertension

- Any cataract that would interfere with OCT

Type of ME: uveitic CME

Age: unknown

Comparability of baseline characteristics: unknown

Interventions: diclofenac and triamcinolone

Test intervention: intravitreal diclofenac and IVTA

Control: none

\section{Outcomes}

Primary outcome: unknown

Measurement of primary outcome: unknown

Secondary outcome: unknown

Measurement of secondary outcome: unknown

Results: unknown

Funding source: unknown

Country: Iran

Masoud Soheilian (2)

Title: To compare therapeutic effect of intravitreal bevacizumab and triamcinolone in resistant uveitic $\mathrm{CME}$ 


\section{Methods}

Study design: randomized interventional parallel-assignment single-blinded (subject)

Method of randomization: unknown

Number of participants: 40

Method of allocation concealment: unknown

Outcome assessor masking: no

Study duration: 24 months

Losses to follow-up: unknown

Intention-to-treat analysis: unknown

\section{Participants}

Inclusion criteria:

- Uveitic CME

- Uveitic CME refractory to routine treatment

- Vision better than 5/200 and worse than 20/50

Exclusion criteria:

- Mono-ocular patients

- History of vitrectomy

- Glaucoma or ocular hypertension

- History of other retinal disease that can cause ME

- Pregnancy

- Significant media opacity

- Vision better than $20 / 50$

Type of ME: uveitic CME

Age: unknown

Comparability of baseline characteristics: unknown

Interventions: Avastin and TA

Test intervention: intravitreal $1.25 \mathrm{mg}$ Avastin and $4 \mathrm{mg}$ IVTA

Control: N/A

\section{Outcomes}

Primary outcome: resorbed CME in OCT (time frame: every 3 months), resorbed CME in clinical examination (time frame: every 3 months)
Measurement of primary outcome: unknown

Secondary outcome: need for retreatment (time frame: whenever needed)

Measurement of secondary outcome: unknown

Results: unknown

Funding source: unknown

Country: Iran

\section{No corresponding author}

Title: The treatment of uveitic CME with topical interferon gamma

\section{Methods}

Study design: nonrandomized, interventional, open-label, single-group assignment

Method of randomization: N/A

Number of participants: 5

Method of allocation concealment: N/A

Outcome assessor masking: no

Study duration: 12 months

Losses to follow-up: unknown

Intention-to-treat analysis: unknown

\section{Participants}

Inclusion criteria:

- Participant must be 18 years of age or older

- Participant must understand and sign the protocol's informed consent document

- Participant has a diagnosis of intermediate, panuveitis, or posterior uveitis at least 3 months prior to study enrollment and has associated CME secondary to uveitis in at least one eye (the study eye)

- Participant has a central macular thickness greater than or equal to 250 microns in the study eye

- Participant is willing to comply with the study procedures and is expected to be able to return for all study visits 
- Participant has VA of $20 / 200$ or better in the study eye

- Female participants of childbearing potential must not be pregnant or breastfeeding

- Both female participants of childbearing potential and male participants able to father a child must agree to practice adequate contraception during the study and for 6 weeks following the administration of study medication. Acceptable methods of contraception include hormonal contraception (ie, birth-control pills, injected hormones, dermal patch, or vaginal ring), intrauterine device, barrier methods with spermicide (diaphragm with spermicide, condom with spermicide) or surgical sterilization (hysterectomy, tubal ligation or vasectomy)

Exclusion criteria:

- Participant is expected to be unable to tolerate the ocular instillation

- Participant is unable to undergo OCT testing

- Participant had herpes keratitis in the past

- Participant is diagnosed with multiple sclerosis

- Participant has a significant active infection (an infection requiring treatment as determined by the medical team) or a history of chronic or recurrent infections that in the principal investigator's best medical judgment would preclude participation

Type of ME: uveitic CME

Age: unknown

Comparability of baseline characteristics: unknown

Interventions: interferon gamma 1-b

Test intervention: topical $10 \mu \mathrm{g}$ interferon gamma 1-b and then increasing dose

Control: N/A

\section{Outcomes}

Primary outcome: change in excess central macular thickening as measured by OCT in response to interferon gamma- $1 \mathrm{~b}$ compared with baseline. Secondary efficacy outcomes include changes in macular volume as measured by OCT, VA, intraocular pressure, and intraocular inflammation as graded upon slit-lamp examination.

Secondary outcome: safety outcomes include ocular surface irritation assessed by fluorescein staining of the cornea and conjunctiva to assess toxicity changes in subjective ocular pain assessments as compared to baseline, number and severity of systemic and ocular toxicities and adverse events, and the proportion of participants with a visual loss of greater than or equal to 15 ETDRS letters

Results: unknown

Funding source: unknown

Country: USA

\section{No corresponding author}

Title: Microplasmin intravitreal administration in participants with uveitic macular edema

Methods

Study design: nonrandomized, interventional, open-label, single-group assignment

Method of randomization: N/A

Number of participants: 5

Method of allocation concealment: N/A

Outcome assessor masking: N/A

Study duration: 18 months

Losses to follow-up: unknown

Intention-to-treat analysis: unknown

\section{Participants}

Inclusion criteria:

- Participant must be 18 years of age or older

- Participant must understand and sign the protocol's informed consent document

- Participant has a diagnosis of uveitic ME that requires treatment in at least one eye (the study eye) and the uveitis in the study eye is deemed clinically quiet by the investigator

- Participant has no evidence of macular or complete posterior vitreous detachment in the study eye by B-scan ultrasound and OCT

- Participant has VA of 20/400 or better in the study eye

- Participant has a central macular thickness greater than or equal to 270 microns in the study eye and loss of the normal foveal contour 
- Participant does not have significant cataract or media opacity in the study eye that makes posterior segment visualization difficult as determined by investigator

- Female participants of childbearing potential must not be pregnant or breastfeeding and must have a negative serum pregnancy test at screening and throughout the study

- Both female participants of childbearing potential and male participants able to father a child must agree to practice two effective methods of birth control for 6 months following administration of study medication. Acceptable methods of birth control for this study include hormonal contraception (birth-control pills, injected hormones, dermal patch, or vaginal ring), intrauterine device, barrier methods (diaphragm, condom) with spermicide or surgical sterilization (hysterectomy, tubal ligation, or vasectomy). Participants with a hysterectomy or vasectomy (or have a partner with a hysterectomy or vasectomy) are exempt from using two methods of birth control

- Participant is willing to comply with the study procedures and return for all study visits

Exclusion criteria:

- Participant has uncontrolled glaucoma, defined as intraocular pressure greater than $30 \mathrm{mmHg}$ despite treatment with antiglaucoma medication, in the study eye

- Participant has lattice degeneration of the retina in the study eye deemed to be high-risk by the investigator

- Participant has untreated retinal holes or tears, or a macular hole in the study eye

- Participant has a significant active ocular infection in the study eye

- Participant had intraocular surgery within the past 90 days or anticipates elective intraocular surgery in the study eye

- Participant had an injection of bevacizumab or ranibizumab within the past 4 weeks in the study eye

- Participant had an injection of triamcinolone within the past 6 weeks in the study eye

- Participant has a condition that in the opinion of the investigator would preclude participation in the study (eg, unstable medical status that would pose a significant hazard if investigational therapy was started)

- Participant has known anaphylaxis to sodium fluoride, or has urticaria, angioedema, or an anaphylactoid response to sodium fluorescein dye that cannot be safely premedicated with an antihistamine and/or prednisone
Type of ME: uveitic CME

Age: unknown

Comparability of baseline characteristics: unknown

Interventions: microplasmin

Test intervention: one-time intravitreal injection of $125 \mu \mathrm{g}$ in $100 \mu \mathrm{L}$ of microplasmin

Control: no

\section{Outcomes}

Primary outcome: safety and tolerability of microplasmin will be assessed by the number and severity of adverse events and systemic and ocular toxicities during the study.

Secondary outcome: potential efficacy of an intravitreal injection of microplasmin for ME secondary to uveitis will be assessed by a change in central macular thickness from baseline measured by OCT in response to microplasmin at 4 and 12 weeks postinjection, the number of participants achieving macular or complete posterior vitreous detachment at 4 and 12 weeks postinjection, the change in ETDRS $\mathrm{BCVA}$ and the change in retinovascular leakage from baseline seen on FA

Results: unknown

Funding source: unknown

Country: USA

\section{No corresponding author}

Title: Treatment of non-infectious intermediate and posterior uveitis associated macular edema with intravitreal methotrexate

\section{Methods}

Study design: nonrandomized, interventional, open-label, single-group assignment

Method of randomization: N/A

Number of participants: 7

Method of allocation concealment: N/A 
Outcome assessor masking: N/A

Study duration: 16 months

Losses to follow-up: unknown

Intention-to-treat analysis: unknown

\section{Participants}

Inclusion criteria:

- Participant must be 18 years of age or older

- Participant must understand and sign the protocol's informed consent document

- Participant is willing to comply with the study procedures and return for all study visits

- Participant has chronic ME secondary to noninfectious panuveitis posterior or intermediate uveitis in at least one eye (the study eye) that has not been responsive to conventional immunosuppressive therapy in the past 3 months and recurred while on conventional immunosuppressive therapy

- Participant has central macular thickness of greater than or equal to 270 microns in the study eye

- Participant has VA of 20/400 or better (greater than or equal to 19 ETDRS letters) in the study eye

- Female participants of childbearing potential must not be pregnant or breastfeeding, must have a negative serum pregnancy test at screening, and must be willing to undergo serum pregnancy tests throughout the study

- Both female participants of childbearing potential and male participants able to father children must have (or have a partner who has) had a hysterectomy or vasectomy, be completely abstinent from intercourse, or must agree to practice two acceptable methods of contraception throughout the course of the study and for 6 months after the last study medication injection. Acceptable methods of contraception include: hormonal contraception (ie, birth-control pills, injected hormones, dermal patch, or vaginal ring), intrauterine device, barrier methods (diaphragm, condom) with spermicide, or surgical sterilization (tubal ligation)

Exclusion criteria:

- Participant is in another investigational study and actively receiving investigational therapy for $\mathrm{ME}$

- Participant has evidence of infectious panuveitis posterior or intermediate uveitis in either eye

- Participant is expected to need ocular surgery in the study eye during the course of the trial
- Participant had intraocular surgery in the study eye within the past 90 days

- Participant had an injection of bevacizumab or ranibizumab within the past 4 weeks in the study eye

- Participant had an injection of triamcinolone within the past 6 weeks in the study eye

- Participant has a systemic condition that in the opinion of the investigator would preclude participation in the study

- Participant has significant cataract or media opacity in the study eye that makes posterior segment visualization difficult as determined by investigator

- Participant has a confirmed positive serologic and/or molecular test for HIV-1/2

Type of ME: uveitic CME

Age: unknown

Comparability of baseline characteristics: unknown

Interventions: methotrexate

Test intervention: intravitreal injections of methotrexate 400 $\mu \mathrm{g} / 100 \mu \mathrm{L}$

Control: N/A

\section{Outcomes}

Primary outcome: number of participants who meet the definition of treatment success within 12 weeks from baseline. Treatment success is defined as achieving at least a 1 -step decrease in the log-score scale for central macular thickness.

Secondary outcome: changes in ETDRS BCVA, changes in excess retinal thickening, changes in macular thickness, changes in intraocular inflammation on clinical exam, changes in leakage as seen on FA, changes in autofluorescence patterns seen on fundus autofluorescence imaging and observation of dose reductions of systemic immunosuppression or steroids. Safety outcomes include the number and severity of adverse events, systemic and ocular toxicities, electrophysiologic changes assessed by full-field electroretinography, and number of withdrawals.

Results: unknown

Funding source: unknown 


\section{Country: USA}

Many corresponding authors, one for each hospital

Title: Efficacy, safety and tolerability of repeated doses of intravitreous bevacizumab in uveitic macular edema

Methods

Study design: randomized uncontrolled open-label clinical trial

Method of randomization: unknown

Number of participants: 60

Method of allocation concealment: N/A

Outcome assessor masking: N/A

Study duration: 72 months

Losses to follow-up: unknown

Intention-to-treat analysis: unknown

\section{Participants}

Inclusion criteria:

- Adult patients with uveitis or retinal vasculitis with unilateral or bilateral $\mathrm{ME}$

- With macular thickness $>250$ microns using OCT

- VA at least 20/200

- With stable treatment with oral prednisone at least during 3 months, either oral cyclosporine or other immunomodulator to treat intraocular inflammatory disease

- Patient that can follow study's requirements

- Patient who consents to participate

Exclusion criteria:

- Presence of corneal or crystalline opacity preventing observation of fundus of eye

- Patients requiring ocular surgery in next 3 months

- One-eyed

- Pregnancy and child breastfeeding

- Previous history of glaucoma

- On treatment with an experimental ocular drug

- Previous thromboembolism or receiving oral anticoagulant treatment

- Vitrectomy

- Patients with proved tractional macular pathology associated to $\mathrm{ME}$
Type of ME: uveitic CME

Age: unknown

Comparability of baseline characteristics: unknown

Interventions: bevacizumab and triamcinolone acetonide

Test intervention: intravitreous bevacizumab $2.5 \mathrm{mg}$ at baseline, week 4 and 8, reinjection if required and IVTA $2 \mathrm{mg}$ at baseline; frequency every 3 months if needed

Control: N/A

\section{Outcomes}

Primary outcome: macular thickness measurements using stratus OCT (time frame: 48 weeks)

Secondary outcome: VA (time frame: 48 weeks), intraocular pressure (time frame: 48 weeks) opacity crystalline (time frame: 48 weeks)

Results: unknown

Funding source: unknown

Country: Spain

\section{Christina J Flaxel}

Title: Lucentis (ranibizumab) for Eales' disease

\section{Methods}

Study design: nonrandomized interventional single-group assignment, open label

Method of randomization: N/A

Number of participants: 5

Method of allocation concealment: N/A

Outcome assessor masking: N/A

Study duration: 30 months

Losses to follow-up: unknown

Intention-to-treat analysis: unknown 


\section{Participants}

Inclusion criteria:

- Ability to provide written informed consent and comply with study assessments for the full duration of the study

- $\quad$ Age $>21$ years

- Disease-related considerations, for both treatment-naïve and previously treated patients:

- exclusion of all other causes of CME and retinal nonperfusion, including branch or central vein occlusion, diabetic retinopathy, sickle retinopathy, sarcoidosis, systemic lupus, and other collagen vascular diseases

- chronic CME as noted clinically and on OCT 3 testing with persistent loss of VA for 3 months or longer

$\circ$ if the eye has received prior treatment (including laser photocoagulation and steroids), a 30-day washout period will be required prior to treatment with Lucentis

- BCVA using ETDRS charts of 20/40 to 20/400 (Snellen equivalent) in the study eye

- $\quad$ OCT 3 central subfield $\geq 250$ on two separate readings in the central subfield

- Only one eye will be assessed in the study. If both eyes are eligible, the investigator will determine which eye will be entered into the study

Exclusion criteria:

- Treatment for ME with intravitreal steroid or Macugen within 30 days prior to enrollment in this study

- Previous vitrectomy within the past 6 months

- Previous cataract surgery within the preceding 12 months

- Active intraocular inflammation in the study eye

- Current vitreous hemorrhage in the study eye

- Active infectious conjunctivitis, keratitis, scleritis, or endophthalmitis in either eye

- A condition that in the opinion of the investigator would preclude participation in the study (eg, unstable medical status, including blood pressure, cardiovascular disease, and glycemic control)

- Participation in an investigational trial within 30 days of randomization that involved treatment with any drug that has not received regulatory approval at the time of study entry

- Known allergy to any component of the study drug

- Blood pressure $>180 / 110$ (systolic above 180 or diastolic above 110)
- If blood pressure is brought below $180 / 110$ by antihypertensive treatment, subject can become eligible

- Major surgery within 28 days prior to randomization or major surgery planned during the next 6 months. Major surgery is defined as a surgical procedure that is more extensive than fine-needle biopsy/aspiration, placement of a central venous access device, removal/biopsy of a skin lesion, or placement of a peripheral venous catheter

- Myocardial infarction, other cardiac event requiring hospitalization, stroke, transient ischemic attack, or treatment for acute congestive heart failure within 6 months prior to randomization

- Systemic anti-VEGF or pro-VEGF treatment within 3 months prior to randomization

- Systemic anti-VEGF or pro-VEGF treatment used during the 6 months of the study

- Current treatment for active systemic infection

- History of recurrent significant infections or bacterial infections

- Subject is expecting to move out of the area of the clinical center to an area not covered by another clinical center during the 6 months of the study

- Subjects meeting any of the following criteria will be excluded from the study:

$\circ$ pregnancy (positive pregnancy test)

$\circ$ prior enrollment in the study

- any other condition that the investigator believes would pose a significant hazard to the subject if the investigational therapy were initiated

- participation in another simultaneous medical investigation or trial

Type of ME: CME secondary to Eale's disease

Age: unknown

Comparability of baseline characteristics: unknown

Interventions: ranibizumab

Test intervention: IV ranibizumab

Control: N/A

Outcomes

Primary outcome:

- Change in OCT thickness (time frame: 6 months) 
- Incidence and severity of ocular adverse events, as identified by eye examination (including VA testing) (time frame: monthly)

- Incidence and severity of other adverse events, as identified by physical examination, subject reporting, and changes in vital signs (time frame: monthly)

Secondary outcome:

- BCVA, as assessed by the number of letters read correctly on the ETDRS eye chart at a starting test distance of 4 meters (time frame: months 3, 4, 5, and 6)
- Incidence of ocular and nonocular adverse events evaluated through month 6 (time frame: monthly)

Results: unknown

Funding source: unknown

Country: USA

\section{Publish your work in this journal}

Clinical Ophthalmology is an international, peer-reviewed journal covering all subspecialties within ophthalmology. Key topics include: Optometry; Visual science; Pharmacology and drug therapy in eye diseases; Basic Sciences; Primary and Secondary eye care; Patient Safety and Quality of Care Improvements. This journal is indexed on

PubMed Central and CAS, and is the official journal of The Society of Clinical Ophthalmology (SCO). The manuscript management system is completely online and includes a very quick and fair peer-review system, which is all easy to use. Visit http://www.dovepress.com/ testimonials.php to read real quotes from published authors. 